\title{
On the solutions of quadratic Diophantine equations II
}

\author{
By Manabu Murata and Takashi YoshinagA
}

(Received Nov. 25, 2016)

\begin{abstract}
A quantity concerning the solutions of a quadratic Diophantine equation in $n$ variables coincides with a mass of a special orthogonal group of a quadratic form in dimension $n-1$, via the mass formula due to Shimura. We show an explicit formula for the quantity, assuming the maximality of a lattice in the $(n-1)$-dimensional quadratic space. The quantity is determined by the computation of a group index and of the mass of the genus of maximal lattices in that quadratic space. As applications of the result, we give the number of primitive solutions for the sum of $n$ squares with 6 or 8 and also the quantity in question for the sum of 10 squares.
\end{abstract}

\section{Introduction.}

We consider an $n$-dimensional vector space $V$ over a totally real number field $F$ and a nondegenerate symmetric $F$-bilinear form $\varphi: V \times V \rightarrow F$, where $2<n \in \mathbf{Z}$. We denote by $\varphi[x]$ the quadratic form $\varphi(x, x)$ on $V$. We assume that $\varphi$ is totally definite. Let $L$ be a maximal lattice in $V$ with respect to $\varphi$, that is, $L$ is a $\mathfrak{g}$-lattice in $V$ which is maximal among $\mathfrak{g}$-lattices on which the values $\varphi[x]$ are contained in $\mathfrak{g}$. Here $\mathfrak{g}$ is the maximal order of $F$. For simplicity, when $\varphi$ is fixed on $V$, we will often refer to a maximal lattice in $V$, omitting reference to the $\varphi$ needed to define it. Put

$$
L[q, \mathfrak{b}]=\{x \in V \mid \varphi[x]=q, \varphi(x, L)=\mathfrak{b}\}
$$

for given $q \in F^{\times}$and fractional ideal $\mathfrak{b}$ of $F$. Assuming $L[q, \mathfrak{b}] \neq \emptyset$, we take an element $h$ of $L[q, \mathfrak{b}]$. Put $W=\{x \in V \mid \varphi(x, h)=0\}$ and let $\psi$ be the restriction of $\varphi$ to $W$. Let $S O^{\psi}(W)$ be the special orthogonal group of $\psi$ and we regard it as the subgroup $\{\alpha \in$ $\left.S O^{\varphi}(V) \mid h \alpha=h\right\}$ of the special orthogonal group $S O^{\varphi}(V)$ of $\varphi$. Here we understand that every $F$-linear automorphism of $V$ acts on $V$ on the right. Put

$$
C(L)=\left\{\gamma \in S O^{\varphi}(V)_{\mathbf{A}} \mid L \gamma=L\right\},
$$

where $S O^{\varphi}(V)_{\mathbf{A}}$ is the adelization of $S O^{\varphi}(V)$ as usual.

It was shown by Shimura in $[\mathbf{7},(3.7)]$ that

$$
\sum_{y \in Y}\left[\Gamma\left(L y^{-1}\right): 1\right]^{-1} \#\left(L y^{-1}\right)[q, \mathfrak{b}]=\mathfrak{m}\left(S O^{\psi}(W), S O^{\psi}(W)_{\mathbf{A}} \cap C(L)\right) .
$$

Here $Y$ is a complete set of representatives for $S O^{\varphi}(V) \backslash S O^{\varphi}(V) \mathbf{A} / C(L), \Gamma\left(L y^{-1}\right)=\{\gamma \in$ $\left.S O^{\varphi}(V) \mid\left(L y^{-1}\right) \gamma=L y^{-1}\right\}$, and $\mathfrak{m}\left(S O^{\psi}(W), S O^{\psi}(W)_{\mathbf{A}} \cap C(L)\right)$ is the mass of $S O^{\psi}(W)$

2010 Mathematics Subject Classification. Primary 11D09; Secondary 11D45, 11E12.

Key Words and Phrases. mass formula, quadratic Diophantine equation, primitive solution. 
relative to the group $S O^{\psi}(W)_{\mathbf{A}} \cap C(L)$; see [7, (3.1)] for the definition. Formula (2) connects the solutions of the equation $\varphi[x]=q$ in $n$ variables, satisfying the condition $\varphi(x, L)=\mathfrak{b}$, with the mass of the group $S O^{\psi}(W)$ in dimension $n-1$. Thus through this mass formula, we are naturally interested in the computation of the right-hand side of $(2)$.

In fact, the purpose of this paper is to give an explicit formula for the mass $\mathfrak{m}\left(S O^{\psi}(W), S O^{\psi}(W)_{\mathbf{A}} \cap C(L)\right)$; see (2.10) and (3.13). To do this, we restrict our investigation to the following case:

The $\mathfrak{g}$-lattice $L \cap W$ in $W$ is maximal with respect to $\psi$.

As for the maximality of $L \cap W$ in $W$, we have one criterion in [10, Theorem 6.3]. That is given as (4.2) in this paper. From this the ideal $\mathfrak{b}$ for which $L \cap W$ is maximal in $W$ is explicitly determined by given $(V, \varphi)$ and $q$.

Here we explain further details about our results. For a moment, we take a maximal lattice $M$ in $W$ and put $E=S O^{\psi}(W)_{\mathbf{A}} \cap C(L)$ for simplicity. By [7, (3.3)] we have $[E: E \cap C(M)] \mathfrak{m}\left(S O^{\psi}(W), E\right)=\mathfrak{m}\left(S O^{\psi}(W), E \cap C(M)\right)=[C(M)$ : $E \cap C(M)] \mathfrak{m}\left(S O^{\psi}(W), C(M)\right)$, where $C(M)=\left\{\gamma \in S O^{\psi}(W)_{\mathbf{A}} \mid M \gamma=M\right\}$. Therefore $\mathfrak{m}\left(S O^{\psi}(W), E\right)$ can be given as follows:

$$
\mathfrak{m}\left(S O^{\psi}(W), E\right)=\frac{[C(M): E \cap C(M)]}{[E: E \cap C(M)]} \mathfrak{m}\left(S O^{\psi}(W), C(M)\right) .
$$

As for the mass $\mathfrak{m}\left(S O^{\psi}(W), C(M)\right)$ of the genus of all maximal lattices in $W$, when the case where the dimension of $W$ over $F$ is even, or the dimension of $W$ is odd and $\operatorname{det}(\psi) \mathfrak{g}$ is a square ideal of $F$, it can be obtained by applying the exact formula due to Shimura [3, Theorem 5.8] to $\mathfrak{m}\left(S O^{\psi}(W), C(M)\right)$, since $M$ is maximal in $W$. When the case where the dimension of $W$ is odd and $\operatorname{det}(\psi) \mathfrak{g}$ is not a square ideal of $F$, a similar formula for $\mathfrak{m}\left(S O^{\psi}(W), C(M)\right)$ is given by [1, Propositions 7.4 and 7.5] due to Gan, Hanke, and Yu. However, we take up again a formula for $\mathfrak{m}\left(S O^{\psi}(W), C(M)\right)$ in the latter case, employing the same ideas in the proof of [3, Theorem 5.8]. By doing so, we can obtain the formula via the determination of a certain group index in Lemma 2.5 below, which is closely connected with the local representation density of (2.9) below at a given prime. In this paper we shall restate the formula in both cases for convenience of the reader (Corollary 2.7).

We now suppose that $L \cap W$ is maximal in $W$. Then we may assume $M=L \cap W$, and hence $E$ is a subgroup of $C(M)$. Therefore we have

$$
\frac{[C(M): E \cap C(M)]}{[E: E \cap C(M)]}=[C(M): E] .
$$

The right-hand side is given by the product of local indices $\left[C\left(M_{v}\right): E_{v}\right]$ for all nonarchimedean primes $v$ of $F$. Here $C\left(M_{v}\right)=\left\{\gamma \in S O^{\psi_{v}}\left(W_{v}\right) \mid M_{v} \gamma=M_{v}\right\}$, $E_{v}=S O^{\psi_{v}}\left(W_{v}\right) \cap C\left(L_{v}\right)$, and $C\left(L_{v}\right)=\left\{\gamma \in S O^{\varphi_{v}}\left(V_{v}\right) \mid L_{v} \gamma=L_{v}\right\}$; see Section 2.1 regarding the symbols $W_{v}, \psi_{v}, M_{v}$, etc. The main result of this paper is to give $\left[C\left(M_{v}\right): E_{v}\right]$ under the assumption that $L_{v} \cap W_{v}$ is maximal in $W_{v}$, where $M_{v}=L_{v} \cap W_{v}$. 
This will be stated in Theorem 3.8. We hope to discuss our investigation on the removal of (3) in the future.

As applications of our results, we give $\# L[q, \mathfrak{b}]$ for a squarefree positive integer $q$ and the ideal $\mathfrak{b}$ such that $L \cap W$ is maximal in $W$, by taking $V=\mathbf{Q}_{n}^{1}(n=6$ or 8$)$, $\varphi[x]=\sum_{i=1}^{n} x_{i}^{2}$ for $x=\left(x_{i}\right)_{i=1}^{n} \in V$, and a maximal lattice $L$ in $V$ (Proposition 4.3 (i) and (ii)). For example, when $n=6$ and $q \equiv 2(\bmod 8)$, we obtain $\# L[q, \mathbf{Z}]=$ $3 \prod_{p \mid q}\left(p^{2}+\left(\frac{-1}{p}\right)\right)$. Here $p$ runs over all prime factors of $q$ and $\left(\frac{-1}{p}\right)$ is the quadratic residue symbol. We also apply the results to the case where $V=\mathbf{Q}_{10}^{1}$ and $\varphi[x]=\sum_{i=1}^{10} x_{i}^{2}$. In this case the class number of $S O^{\varphi}(V)$ with respect to $C(L)$ is 2 (cf. [2, Section 3.2] due to Hiraoka). Let $\left\{L_{1}, L_{2}\right\}$ be a complete set of representatives for the $S O^{\varphi}(V)$-classes in the genus of all maximal lattices in $V$; see [6, Section 9.3] for the definition of the $S O^{\varphi}(V)$-class. An explicit choice of each $L_{i}(i=1,2)$ is given in (4.3). Then we give $\sum_{i=1}^{2}\left[\Gamma\left(L_{i}\right): 1\right]^{-1} \# L_{i}[q, \mathfrak{b}]$ for a squarefree positive integer $q$ (Proposition 4.3 (iii)). For example, when $q \equiv 1,2(\bmod 4)$, we have

$$
\sum_{i=1}^{2} \frac{\# L_{i}[q, \mathbf{Z}]}{\left[\Gamma\left(L_{i}\right): 1\right]}=\frac{1}{2^{19} \cdot 3^{5} \cdot 5^{2} \cdot 7} \prod_{p \mid q}\left(p^{4}+\left(\frac{-1}{p}\right)\right) \cdot\left\{\begin{array}{lll}
2^{5} & \text { if } q \equiv 1 & (\bmod 4) \\
17 & \text { if } q \equiv 2 \quad(\bmod 8) \\
3 \cdot 5 & \text { if } q \equiv 6 & (\bmod 8)
\end{array}\right.
$$

Moreover, a few numerical computation in both sides of above equality is given after Proposition 4.3. In Section 4.1 we will explain the relationship between the set $L[q, \mathfrak{b}]$ and a set of primitive solutions of a quadratic Diophantine equation.

Acknowledgements. The authors would like to express their deepest gratitude to Professor Koji Doi who gave them several useful comments throughout this investigation and enthusiastically recommended them to add the applications in the paper; and to Dr. Yoshio Hiraoka who computed (by computer) the primitive solutions of quadratic forms in Section 4 by taking more than 500 cases in " $q$ ". The first author would like to thank Dr. Seidai Yasuda who outlined a fact on primitive solutions involving the Möbius function after the seminar at Osaka University on July 10, 2015.

Notations. We follow the notion and the notation in Shimura's book $[6]$ and our previous paper $[\mathbf{1 0}]$.

If $X$ is a set, then $\# X$ denotes the cardinality of $X$. If $X$ is a disjoint union of its subsets $Y_{1}, \cdots, Y_{m}$, we write $X=\bigsqcup_{i=1}^{m} Y_{i}$ or $X=Y_{1} \sqcup \cdots \sqcup Y_{m}$. For a subgroup $H$ of a group $G$ we let $[G: H]=\#(H \backslash G)$. For a real number $a$ we denote by $[a]$ the greatest integer not greater than $a$.

If $R$ is an associative ring with identity element, then $R^{\times}$is the group of units of $R$. Put $R^{\times 2}=\left\{a^{2} \mid a \in R^{\times}\right\}$. If $K$ is a finite algebraic extension of a field $F$, then $D_{K / F}$ denotes the relative discriminant of $K$ over $F$.

Let $F$ be a number field or its completion, and $V$ an $n$-dimensional vector space over $F$. By a $\mathfrak{g}$-lattice in $V$, we understand a finitely generated $\mathfrak{g}$-module in $V$ that spans $V$ over $F$. Here $\mathfrak{g}$ is the maximal order of $F$. We write $\operatorname{dim}_{F}(V)$ for the dimension of $V$ over $F$. We denote by $1_{V}$ the identity map of $V$. If $R=F$ or $\mathfrak{g}$, then we write $R_{n}^{m}$ for the $R$-module of all $m \times n$-matrices with entries in $R$. We denote by 0 the zero element 
of $R_{n}^{m}$. Put $M_{n}(R)=R_{n}^{n}$ when we regard $R_{n}^{n}$ as a ring. We let $1_{n}$ denote the identity element of $M_{n}(R)$. Put $G L_{n}(R)=M_{n}(R)^{\times}$. For a matrix $x$ we denote by ${ }^{t} x, \operatorname{det}(x)$, and $\operatorname{tr}(x)$ the transpose, determinant, and trace of $x$. Put $N(\mathfrak{a})=N \mathfrak{a}=[\mathfrak{g}: \mathfrak{a}]$ for an integral ideal $\mathfrak{a}$ of $\mathfrak{g}$.

\section{The mass $\mathfrak{m}\left(S O^{\psi}(W), C(M)\right)$.}

2.1. For a given quadratic space $(V, \varphi)$ over $F$ equipped with a totally-definite symmetric $F$-bilinear form $\varphi$, we assume that

the dimension of $V$ is odd and $\operatorname{det}(\varphi) \mathfrak{g}$ is not a square ideal of $F$.

We will determine the mass of the genus of all maximal lattices in the quadratic space $(V, \varphi)$. Once a formula for the mass is obtained, the formula is applicable to $\mathfrak{m}\left(S O^{\psi}(W), C(M)\right)$ in (4) if $(W, \psi)$ satisfies $(2.1)$. Here $M=L \cap W$ is a maximal lattice in $W$. When the case where the dimension of $W$ over $F$ is even, or the dimension of $W$ is odd and $\operatorname{det}(\psi) \mathfrak{g}$ is a square ideal of $F$, the formula in [3, Theorem 5.8] is applicable to $\mathfrak{m}\left(S O^{\psi}(W), C(M)\right)$ in question.

Let $L$ be a maximal lattice in $V$ and define $C(L)$ by (1). Let $\varphi_{v}$ be the $F_{v}$-linear extension of $\varphi$ to $V_{v}=V \otimes_{F} F_{v}$ and put $L_{v}=L \otimes_{\mathfrak{g}} \mathfrak{g}_{v}$ for $v \in \boldsymbol{h}$. Here $F_{v}$ is the $v$-completion of $F, \mathfrak{g}_{v}$ is the maximal order of $F_{v}$, and $\boldsymbol{h}$ is the set of all nonarchimedean primes of $F$. Put $\widetilde{L}=\{x \in V \mid 2 \varphi(x, L) \subset \mathfrak{g}\}$. Let $\mathfrak{e}$ be the product of all prime ideals for which $\widetilde{L_{v}} \neq L_{v}$.

First, in the same manner as in [3, Section 7.10], we obtain

$$
\mathfrak{m}\left(S O^{\varphi}(V), C(L)\right)=\mathfrak{m}\left(S O^{\varphi}(V), D(\mathfrak{a})\right) \prod_{v \mid \mathfrak{a}} \frac{2}{\left[D\left(L_{v}\right): D_{v}^{\varphi}\right]}
$$

for an integral ideal $\mathfrak{a}$ of $\mathfrak{g}$ such that $\mathfrak{a} \subset \mathfrak{e}$ and $2 \notin \mathfrak{a}_{v}$ for every $v \mid \mathfrak{a}$. Here $D(\mathfrak{a})=$ $\left\{\gamma \in C(L) \mid \widetilde{L_{v}}\left(\gamma_{v}-1\right) \subset \mathfrak{a}_{v} L_{v}\right.$ for every $\left.v \mid \mathfrak{a}\right\}, D\left(L_{v}\right)=\left\{\gamma \in O^{\varphi_{v}}\left(V_{v}\right) \mid L_{v} \gamma=L_{v}\right\}$, and $D_{v}^{\varphi}=\left\{\gamma \in D\left(L_{v}\right) \mid \widetilde{L_{v}}(\gamma-1) \subset \mathfrak{a}_{v} L_{v}\right\}$.

The mass $\mathfrak{m}\left(S O^{\varphi}(V), D(\mathfrak{a})\right)$ in $(2.2)$ is given as follows:

$$
\begin{gathered}
\mathfrak{m}\left(S O^{\varphi}(V), D(\mathfrak{a})\right)=\mathfrak{m}_{n}(\mathfrak{a})[\widetilde{L}: L]^{(n-1) / 2} \\
\mathfrak{m}_{n}(\mathfrak{a})=2^{1-g(n-1) / 2} N(\mathfrak{a})^{n(n-1) / 2} D_{F}^{\left[(n-1)^{2} / 4\right]} \prod_{i=1}^{[(n-1) / 2]}\left\{D_{F}^{1 / 2}\left[(2 i-1) !(2 \pi)^{-2 i}\right]^{g} \zeta_{F, \mathfrak{a}}(2 i)\right\} .
\end{gathered}
$$

Here $g=[F: \mathbf{Q}], D_{F}$ is the discriminant of $F, \zeta_{F, \mathfrak{a}}(s)=\zeta_{F}(s) \prod_{v \mid \mathfrak{a}}\left(1-N\left(\mathfrak{p}_{v}\right)^{-s}\right)$, $\zeta_{F}$ is the Dedekind zeta function of $F$, and $\mathfrak{p}_{v}$ is the prime ideal of $\mathfrak{g}$ corresponding to $v$. Formula $(2.3)$ can be obtained in exactly the same manner as in the proof of $[\mathbf{3}$, Theorem 5.8], more precisely, as in the argument in [3, Section 7].

2.2. We are going to compute the index $\left[D\left(L_{v}\right): D_{v}^{\varphi}\right]$ in $(2.2)$ for $v \mid \mathfrak{a}$. We fix such a $v$ and drop the subscript $v$ for simplicity, until the end of the proof of Lemma 2.5. 
Let $\pi$ be a prime element of $\mathfrak{g}$ and $\mathfrak{p}$ the prime ideal of $\mathfrak{g}$. From assumption (2.1) we may assume that

$$
\operatorname{dim}_{F}(V)=n \notin 2 \mathbf{Z} \text { and } \operatorname{det}(\varphi) \in \pi \mathfrak{g}^{\times} F^{\times 2}
$$

Otherwise the desired index is given by [3, Proposition 3.9].

Put $D_{i}=D_{i}^{\varphi}=\left\{\gamma \in D(L) \mid \widetilde{L}(\gamma-1) \subset \mathfrak{p}^{i} L\right\}$ for $0<i \in \mathbf{Z}$. Then we have $D^{\varphi}=D_{\nu}$ with $\nu \in \mathbf{Z}$ such that $\mathfrak{a}=\mathfrak{p}^{\nu}$. It can be seen that $\left[D(L): D^{\varphi}\right]=[D(L)$ : $\left.D_{1}\right] \prod_{i=1}^{\nu-1}\left[D_{i}: D_{i+1}\right]$. The same argument as in the proof of [3, Lemma 8.5] gives $\left[D_{i}: D_{i+1}\right]=q^{n(n-1) / 2}$, where $q=[\mathfrak{g}: \mathfrak{p}]$. Thus we obtain

$$
\left[D(L): D^{\varphi}\right]=\left[D(L): D_{1}\right] q^{(\nu-1) n(n-1) / 2} .
$$

2.3. To determine the index $\left[D(L): D_{1}\right]$ in $(2.5)$, let us recall a Witt decomposition of $V$ with respect to $\varphi$ as follows (cf. [6, Lemma 6.5]):

$$
V=Z+\sum_{i=1}^{r}\left(F e_{i}+F f_{i}\right), \quad L=N+\sum_{i=1}^{r}\left(\mathfrak{g} e_{i}+\mathfrak{g} f_{i}\right) .
$$

Here $e_{i}$ and $f_{i}$ are elements of $V$ such that $\varphi\left(e_{i}, e_{j}\right)=\varphi\left(f_{i}, f_{j}\right)=0$ and $\varphi\left(e_{i}, f_{j}\right)=2^{-1} \delta_{i j}$, $Z=\left\{x \in V \mid \varphi\left(e_{i}, x\right)=\varphi\left(f_{i}, x\right)=0\right.$ for every $\left.i\right\}, N=\{x \in Z \mid \varphi[x] \in \mathfrak{g}\}$, and the restriction $\theta$ of $\varphi$ to $Z$ is anisotropic. Then we say that $Z$ is a core subspace of $V$ with respect to $\varphi$. By $[6$, Lemma 6.4], note that $N$ is a unique maximal lattice in $Z$, and therefore $O^{\theta}(Z)=D(N)$. Put $t=\operatorname{dim}_{F}(Z)$, then $n=2 r+t$. We call $t$ the core dimension of $(V, \varphi)$. We have $t \leq 4$ by [6, Theorem 7.6 (ii)]. We note that $t=1$ or 3 in our assumption (2.4). Hereafter we fix such a decomposition until the end of the proof of Lemma 2.5 .

Let us further recall some basic facts on the core subspace $Z$ of dimension $t \leq 4$ over $F$, following [6, Section 7], which will be needed in our determination and in the next section. For an element $q$ of $\mathfrak{g}$ we put $N[q]=\{z \in N \mid \theta[z]=q\}$. Throughout this paper, we denote by $A(V)$ the Clifford algebra of $\varphi$ for a quadratic space $(V, \varphi)$ and by $A^{+}(V)$ its even Clifford algebra.

If $t=1$, then $Z=F g$ with some $g \in Z$. We identify $Z$ with $F$ via the map of $Z$ onto $F$ defined by $a g \mapsto a$. Then, putting $c=\theta[g]$, we have $\theta[a]=c a^{2}$ for $a \in F$. Let $c \mathfrak{g}=\mathfrak{p}^{\varepsilon}$. We see that $N=\mathfrak{p}^{-[\varepsilon / 2]}$. Clearly $N[q]=\emptyset$ if and only if $c q \notin F^{\times 2}$.

Assume $t=2$. Let $Z=F g+F k$ with some elements $g$ and $k$ such that $\theta(g, k)=0$. We consider the even Clifford algebra $A^{+}(Z)$ of $\theta$ and put $K=A^{+}(Z)$. Then via the map $a g \mapsto a,(Z, \theta)$ is isomorphic to $(K, c \kappa)$, where $\kappa$ is the norm form on $K$ and $c=\theta[g]$. Put $c \mathfrak{g}=\mathfrak{p}^{\varepsilon}$. Note that $K$ is a quadratic extension of $F$, which gives the discriminant field $F\left([-\operatorname{det}(\theta)]^{1 / 2}\right)$ of $(Z, \theta)$. We identify $(Z, \theta)$ with $(K, c \kappa)$ through the isomorphism. If $K$ is ramified over $F$, then we can take $c$ so that $c \in \mathfrak{g}^{\times} F^{\times 2}$. Then $N=\mathfrak{q}^{-\varepsilon}$, where $\mathfrak{q}$ is the prime ideal of the maximal order $\mathfrak{r}$ of $K$. If $K$ is unramified over $F$, then $N=\mathfrak{p}^{-[\varepsilon / 2]} \mathfrak{r}$. Clearly $N[q]=\emptyset$ if and only if $q \notin c \kappa\left[K^{\times}\right]$. Also by [8, Theorem 20.8 (ii)], $c \in \kappa\left[K^{\times}\right]$if and only if the Clifford algebra $A(Z)$ of $\theta$ is isomorphic to $M_{2}(F)$.

If $t=3$, then $A^{+}(Z)$ is a division quaternion algebra $B$ over $F$. Put $B^{\circ}=\{x \in$ 
$\left.B \mid x+x^{\iota}=0\right\}$ with the main involution $\iota$ of $B$ and let $\beta^{\circ}$ be the restriction of the norm form $\beta$ on $B$ to $B^{\circ}$. There is an element $\zeta$ of $A(Z)^{\times}$such that $Z=B^{\circ} \zeta$. Thus $(Z, \theta)$ is isomorphic to $\left(B^{\circ}, d \beta^{\circ}\right)$ via the map defined by $x \mapsto x \zeta^{-1}$, where $d$ is an element of $F^{\times}$ such that $d F^{\times 2}=\operatorname{det}(\theta) F^{\times 2}$. Put $d \mathfrak{g}=\mathfrak{p}^{\varepsilon}$. We identify $(Z, \theta)$ with $\left(B^{\circ}, d \beta^{\circ}\right)$ through the isomorphism. Then we have $B=J+J \omega$ with an unramified quadratic extension $J$ of $F$ and an element $\omega$ of $B^{\circ}$ such that $\omega^{2} \in \pi \mathfrak{g}^{\times}$and $a \omega=\omega a^{\iota}$ for $a \in J$. Take an element $u$ of $J$ such that $\mathfrak{r}=\mathfrak{g}[u]$ and $u-u^{\iota} \in \mathfrak{r}^{\times}$, where $\mathfrak{r}$ is the maximal order of $J$. Then $Z=F \eta+J \omega$ and $N=\mathfrak{p}^{-\varepsilon / 2}(\mathfrak{g} \eta+\mathfrak{r} \omega)$ or $N=\mathfrak{p}^{-(\varepsilon-1) / 2}\left(\mathfrak{g} \eta+\mathfrak{r} \omega^{-1}\right)$ according as $d \in \mathfrak{g}^{\times} F^{\times 2}$ or $d \in \pi \mathfrak{g}^{\times} F^{\times 2}$, where $\eta=u-u^{\iota}$. Note by [6, Proposition 5.15 (ii)] that $N[q]=\emptyset$ if and only if $-d q \in F^{\times 2}$. Even if $\theta$ is isotropic, we can employ the setting similar to the anisotropic case. Namely, taking $B=M_{2}(F), \beta[x]=\operatorname{det}(x)$, and $B^{\circ}=\left\{x \in M_{2}(F) \mid \operatorname{tr}(x)=0\right\}$, we regard $(Z, \theta)$ as $\left(B^{\circ}, d \beta^{\circ}\right)$ with $d \in F^{\times}$.

Assume $t=4$. Then by [6, Theorem 7.5 (ii)], we may identify $(Z, \theta)$ with $(B, \beta)$ with a division quaternion algebra $B$ over $F$ and the norm form $\beta$ on $B$. Then $N$ is the maximal order of $B$. We note that $\operatorname{det}(\theta) \in F^{\times 2}$.

Lemma 2.4. Assume (2.4). Then

$$
\left[D(N): D_{1}^{\theta}\right]= \begin{cases}2 & \text { if } t=1, \\ 4 q^{2}(q+1) & \text { if } t=3 .\end{cases}
$$

Here $D_{1}^{\theta}$ is defined in a similar way to $D_{1}^{\varphi}$ with $N$ in place of $L$.

Proof. Assume $t=1$. Then $Z=F$ and $\theta[a]=c a^{2}$ with $c \in \pi \mathfrak{g}^{\times}$, since $\varphi$ satisfies (2.4). We have $D(N)=O^{\theta}(Z)=\{ \pm 1\}$. Because $N=\mathfrak{g}$, clearly $\widetilde{N}=(2 \mathfrak{p})^{-1}$. Hence we obtain $D_{1}^{\theta}=\{1\}$. Thus $\left[D(N): D_{1}^{\theta}\right]=2$.

Assume $t=3$. Then $Z=F \eta+J \omega, \theta[z]=d \beta^{\circ}[z]$, and $N=\mathfrak{g} \eta+\mathfrak{r} \omega^{-1}$ with $d \in \pi \mathfrak{g}^{\times}$ by (2.4). We see that $\widetilde{N}=(2 \mathfrak{p})^{-1} \eta+\mathfrak{r} \omega^{-1}$. For $a \in B$, let $\tau_{a}$ be an $F$-linear automorphism of $Z$ defined by $x \tau_{a}=a^{-1} x a$ for $x \in Z$. Put $C=\left\{\tau_{a} \mid a \in \mathfrak{O}^{\times}\right\}$, where $\mathfrak{O}$ is the maximal order of $B$. Then, in a similar way to the proof of [3, Lemma 3.7], we can show that $D(N)=C \sqcup\left(-1_{Z}\right) C \sqcup \tau_{\omega} C \sqcup\left(-1_{Z}\right) \tau_{\omega} C, D_{1}^{\theta} \subset C$, and $D_{1}^{\theta}=\left\{\tau_{a} \mid a \in \mathfrak{g}^{\times}\left(1+\mathfrak{P}^{2}\right)\right\}$, where $\mathfrak{P}$ is the prime ideal of $\mathfrak{O}$. Thus we obtain $\left[D(N): D_{1}^{\theta}\right]=4\left[C: D_{1}^{\theta}\right]=4 q^{2}(q+1)$. This completes the proof.

Throughout this paper, for a subspace $X$ of a given quadratic space $(V, \varphi)$ we put

$$
X^{\perp}=\{y \in V \mid \varphi(y, X)=\{0\}\} .
$$

Lemma 2.5. Assume $(2.4) ;$ put $\ell=(n-1) / 2$ and $k=n(n-1) / 2$. Then

$$
\left[D(L): D_{\nu}^{\varphi}\right]=2 q^{\nu k} \cdot \begin{cases}2\left(1-q^{-\ell}\right) \prod_{i=1}^{\ell-1}\left(1-q^{-2 i}\right) & \text { if } t=1, \\ 2\left(1+q^{-\ell}\right) \prod_{i=1}^{\ell-1}\left(1-q^{-2 i}\right) & \text { if } t=3\end{cases}
$$

for $\nu$ as in $(2.5)$.

When (2.4) is not satisfied, the index $\left[D(L): D_{\nu}^{\varphi}\right]$ is given by [3, Proposition 3.9]. 
Proof. By (2.5) it is enough to determine $\left[D(L): D_{1}\right]$. If $r=0$, then this index is given by Lemma 2.4. Assume $r>0$. Put $W=\left(F e_{1}+F f_{1}\right)^{\perp}$ and let $\sigma$ be the restriction of $\varphi$ to $W$. Put $M=L \cap W$. Then we shall show that

$$
\left[D(L): D_{1}^{\varphi}\right]=q^{n-2} A_{0}\left[D(M): D_{1}^{\sigma}\right]
$$

with the value $A_{0}$ given as follows:

$$
A_{0}= \begin{cases}q\left(q^{\ell}-1\right)\left(q^{\ell-1}+1\right) & \text { if } t=1, \\ q\left(q^{\ell}+1\right)\left(q^{\ell-1}-1\right) & \text { if } t=3 .\end{cases}
$$

If $\sigma$ is anisotropic on $W$, then (2.7) together with Lemma 2.4 gives the index $\left[D(L): D_{1}^{\varphi}\right]$. If $\sigma$ is isotropic on $W,(2.7)$ is applicable to $\left[D(M): D_{1}^{\sigma}\right]$ instead of $\left[D(L): D_{1}^{\varphi}\right]$. Repeating this process, we obtain $\left[D(L): D_{1}^{\varphi}\right]$.

The proof of $(2.7)$ can be seen in a similar way to [3, Section 3] in view of the difference between the present form $\varphi$ and the quadratic form in [3, Section 3], which does not satisfy (2.4). Let us give an outline of the proof.

Put $C^{\prime}=\left\{\alpha \in D(L) \mid e_{1} \alpha-e_{1} \in \mathfrak{p} L, f_{1} \alpha-f_{1} \in \mathfrak{p} L\right\}$. Then $D_{1}^{\varphi} \subset C^{\prime} \subset D(L)$, and hence $\left[D(L): D_{1}^{\varphi}\right]=\left[D(L): C^{\prime}\right]\left[C^{\prime}: D_{1}^{\varphi}\right]$. It can be seen that $\left[C^{\prime}: D_{1}^{\varphi}\right]=\left[D(M): D_{1}^{\sigma}\right]$ in the same manner as in the proof of [3, Proposition 3.9]. Furthermore we see that $\left[D(L): C^{\prime}\right]=q^{n-2} \# Y_{0}$, where $Y_{0}=\{x+\mathfrak{p} L \in L / \mathfrak{p} L \mid x \notin \mathfrak{p} L, \varphi[x]=0\}$. Then we have

$$
\# Y_{0}=\# Y-q \text {, }
$$

where $Y=\{x+\mathfrak{p} L \in L / \mathfrak{p} L \mid \varphi[x] \in \mathfrak{p}\}$. To see (2.8), suppose $t=3$. Then $Z=$ $F \eta+J \omega, \theta[z]=d \beta^{\circ}[z]$, and $N=\mathfrak{g} \eta+\mathfrak{r} \omega^{-1}$ with $d \in \pi \mathfrak{g}^{\times}$by (2.4). By employing this setting, the argument similar to $\left[\mathbf{3}\right.$, Lemma 3.8] shows $\# Y-\# Y_{0}=\#\left\{a \eta+b \omega^{-1}+\right.$ $\mathfrak{p} N \in N / \mathfrak{p} N \mid a \in \mathfrak{g}, b \in \mathfrak{p r}\}$. Hence we have (2.8). Still assuming $t=3$, put $I(\varphi)=$ $\sum_{x \in L / \mathfrak{p} L} \sum_{\xi \in \mathfrak{g} / \mathfrak{p}} \chi(\xi \varphi[x] / \pi)$. Here $\chi$ is a $\mathbf{T}$-valued character of the additive group $F$ such that $\mathfrak{g}=\{a \in F \mid \chi(a \mathfrak{g})=1\}$, where $\mathbf{T}=\{z \in \mathbf{C}|| z \mid=1\}$. We see that $I(\varphi)=\# Y \cdot q$. The equality $I(\varphi)=q^{n}-q^{r+t}+q^{r} I(\theta)$ is valid even for the present $\varphi$, where $I(\theta)$ is defined in a similar way to $I(\varphi)$ with $N$ and $\theta$ in place of $L$ and $\varphi$. We then find that $I(\theta)=q^{2}$. To sum up, we have $\left[D(L): C^{\prime}\right]=q^{n-2} A_{0}$ with $A_{0}$ in the statement in the case $t=3$. Similarly we obtain the assertion in the case $t=1$. This proves (2.7), which completes the proof.

The index $\left[D(L): D_{\nu}^{\varphi}\right]$ in Lemma 2.5 is connected with a local representation density. In fact, by $(2.5)$ and $\left[\mathbf{3}\right.$, Theorem 8.6] we have $\left[D(L): D_{\nu}^{\varphi}\right]=q^{\nu k-\kappa n}[\widetilde{L}: L]^{-1} e\left(2 \varphi_{0}\right)$, where $2 \mathfrak{g}=\mathfrak{p}^{\kappa}$ and

$$
e\left(2 \varphi_{0}\right)=\lim _{m \rightarrow \infty} q^{-m k} \cdot \#\left\{y \in \mathfrak{g}_{n}^{n} /\left.\pi^{m} \mathfrak{g}_{n}^{n}\right|^{t} y\left(2 \varphi_{0}\right) y-2 \varphi_{0} \in \pi^{m} \mathfrak{g}_{n}^{n}\right\} .
$$

Here $\varphi_{0}$ is the matrix that represents $\varphi$ with respect to a $\mathfrak{g}$-basis of $L$.

We are now ready to state the formula for $\mathfrak{m}\left(S O^{\varphi}(V), C(L)\right)$ containing the case where $\varphi$ does not satisfy $(2.1)$. 
Proposition 2.6. Let $V$ be an $n$-dimensional vector space over a totally real number field $F$ and $\varphi$ a totally-definite symmetric $F$-bilinear form on $V$, where $n$ is an arbitrary integer greater than 1 . Let $L$ be a maximal lattice in $V$ with respect to $\varphi$. Put $C(L)=\left\{\gamma \in S O^{\varphi}(V)_{\mathbf{A}} \mid L \gamma=L\right\}$ and

$$
\begin{aligned}
\mathfrak{m}_{n}(\mathfrak{g}) & =2 D_{F}^{\left[(n-1)^{2} / 4\right]} \prod_{i=1}^{[(n-1) / 2]}\left\{D_{F}^{1 / 2}\left[(2 i-1) !(2 \pi)^{-2 i}\right]^{g} \zeta_{F}(2 i)\right\} \\
& \cdot \begin{cases}2^{-g(n-1) / 2} & \text { if } n \text { is odd }, \\
N(\mathfrak{d})^{1 / 2} D_{F}^{1 / 2}\left[(m-1) !(2 \pi)^{-m}\right]^{g} L(m, \sigma) & \text { if } n \text { is even } .\end{cases}
\end{aligned}
$$

Here $D_{F}$ is the discriminant of $F, g=[F: \mathbf{Q}], m=n / 2$, and $\mathfrak{d}$ is the different of $F\left(\left[(-1)^{m} \operatorname{det}(\varphi)\right]^{1 / 2}\right)$ relative to $F ; \zeta_{F}$ is the Dedekind zeta function of $F$ and $L(s, \sigma)$ is the L-function of the Hecke character $\sigma$ of $F$ corresponding to the extension $F\left(\left[(-1)^{m} \operatorname{det}(\varphi)\right]^{1 / 2}\right) / F$. Then

$$
\mathfrak{m}\left(S O^{\varphi}(V), C(L)\right)=\mathfrak{m}_{n}(\mathfrak{g})[\widetilde{L}: L]^{(n-1) / 2} \prod_{v \mid \mathfrak{e}} \lambda_{v} \cdot \begin{cases}1 & \text { if } n \text { is odd, } \\ N(\mathfrak{d})^{-1 / 2} & \text { if } n \text { is even }\end{cases}
$$

with the value $\lambda_{v}$ given as follows:

$$
\begin{cases}1 & \text { if } t_{v}=1, v \mid 2, \text { and } \operatorname{det}(\varphi) \in \mathfrak{g}_{v}^{\times} F_{v}^{\times 2}, \\ 2^{-1}\left(1+q_{v}^{-(n-1) / 2}\right) & \text { if } t_{v}=1 \text { and } \operatorname{det}(\varphi) \in \pi_{v} \mathfrak{g}_{v}^{\times} F_{v}^{\times 2}, \\ 2^{-1}\left(1+q_{v}\right)^{-1}\left(1+q_{v}^{1-m}\right)\left(1+q_{v}^{-m}\right) & \text { if } t_{v}=2, \mathfrak{d}_{v}=\mathfrak{r}_{v}, \text { and } \widetilde{N}_{v} \neq N_{v}, \\ 2^{-1} & \text { if } t_{v}=2 \text { and } \mathfrak{d}_{v} \neq \mathfrak{r}_{v}, \\ 2^{-1}\left(1+q_{v}\right)^{-1}\left(1-q_{v}^{1-n}\right) & \text { if } t_{v}=3 \text { and } \operatorname{det}(\varphi) \in \mathfrak{g}_{v}^{\times} F_{v}^{\times 2}, \\ 2^{-1}\left(1-q_{v}^{-(n-1) / 2}\right) & \text { if } t_{v}=3 \text { and } \operatorname{det}(\varphi) \in \pi_{v} \mathfrak{g}_{v}^{\times} F_{v}^{\times 2}, \\ 2^{-1}\left(1+q_{v}\right)^{-1}\left(1-q_{v}^{1-m}\right)\left(1-q_{v}^{-m}\right) & \text { if } t_{v}=4 .\end{cases}
$$

Here $\widetilde{L}=\{x \in V \mid 2 \varphi(x, L) \subset \mathfrak{g}\}, \mathfrak{e}$ is the product of all prime ideals for which $\widetilde{L_{v}} \neq L_{v}$, and $\mathfrak{r}$ is the maximal order of $F\left(\left[(-1)^{m} \operatorname{det}(\varphi)\right]^{1 / 2}\right) ; t_{v}$ is the core dimension of $\left(V_{v}, \varphi_{v}\right)$, $\widetilde{N_{v}}=\left\{x \in Z_{v} \mid 2 \varphi_{v}\left(x, N_{v}\right) \subset \mathfrak{g}_{v}\right\}, N_{v}$ and $Z_{v}$ are as in (2.6), and $q_{v}$ is the norm of the prime ideal of $\mathfrak{g}_{v}$.

We note that $v \nmid \mathfrak{e}$ if $t_{v}=0$, if $t_{v}=1, v \nmid 2$, and $\operatorname{det}(\varphi) \in \mathfrak{g}_{v}^{\times} F_{v}^{\times 2}$, or if $t_{v}=2$, $\mathfrak{d}_{v}=\mathfrak{r}_{v}$, and $\widetilde{N_{v}}=N_{v}$. Also note that $F\left(\left[(-1)^{n / 2} \operatorname{det}(\varphi)\right]^{1 / 2}\right)$ is a quadratic extension of $F$ if $t_{v}=2$.

Proof. When $\varphi$ does not satisfy (2.1), the formula is nothing but the exact formula given in [3, Theorem 5.8]. When $\varphi$ satisfies (2.1), there exists $v \in \boldsymbol{h}$ satisfying (2.4). For such a $v,\left[D\left(L_{v}\right): D_{v}^{\varphi}\right]$ in (2.2) is given by Lemma 2.5. If $v$ does not satisfy (2.4), then [3, Proposition 3.9] gives $\left[D\left(L_{v}\right): D_{v}^{\varphi}\right]$. Combining this with $(2.2)$ and $(2.3)$, we obtain the desired formula. 
By applying Proposition 2.6 to $\mathfrak{m}\left(S O^{\psi}(W), C(M)\right)$ in (4) in the introduction, we obtain the following formula for this mass:

COROLlary 2.7. Let $V$ be an $n$-dimensional vector space over a totally real number field $F$ and $\varphi$ a totally-definite symmetric $F$-bilinear form on $V$, where $2<n \in \mathbf{Z}$. Let $L$ be a maximal lattice in $V$ with respect to $\varphi$. Assume $L[q, \mathfrak{b}] \neq \emptyset$ with $q \in F^{\times}$and a fractional ideal $\mathfrak{b}$ of $F$. For an element $h$ of $L[q, \mathfrak{b}]$, put $W=(F h)^{\perp}$ and let $\psi$ be the restriction of $\varphi$ to $W$. We regard $S O^{\psi}(W)$ as the subgroup $\left\{\alpha \in S O^{\varphi}(V) \mid h \alpha=h\right\}$ of $S O^{\varphi}(V)$. Assume that the $\mathfrak{g}$-lattice $L \cap W$ is maximal in $W$. Put $M=L \cap W$ and $C(M)=\left\{\gamma \in S O^{\psi}(W)_{\mathbf{A}} \mid M \gamma=M\right\}$. Also we put

$$
\begin{aligned}
\mathfrak{m}_{n-1}(\mathfrak{g}) & =2 D_{F}^{\left[(n-2)^{2} / 4\right]} \prod_{i=1}^{[(n-2) / 2]}\left\{D_{F}^{1 / 2}\left[(2 i-1) !(2 \pi)^{-2 i}\right]^{g} \zeta_{F}(2 i)\right\} \\
& \cdot \begin{cases}2^{-g(n-2) / 2} & \text { if } n \text { is even }, \\
N\left(\mathfrak{d}_{K}\right)^{1 / 2} D_{F}^{1 / 2}\left[(\ell-1) !(2 \pi)^{-\ell}\right]^{g} L\left(\ell, \sigma_{K}\right) & \text { if } n \text { is odd } .\end{cases}
\end{aligned}
$$

Here $K=F\left(\sqrt{(-1)^{n-1} \delta q}\right) / F, \delta=(-1)^{n(n-1) / 2} \operatorname{det}(\varphi), \mathfrak{d}_{K}$ is the different of $K$ relative to $F, \ell=(n-1) / 2$, and $L\left(s, \sigma_{K}\right)$ is the L-function of the Hecke character $\sigma_{K}$ of $F$ corresponding to the extension $K / F ; D_{F}, g$, and $\zeta_{F}$ are as in Proposition 2.6. Then

$$
\mathfrak{m}\left(S O^{\psi}(W), C(M)\right)=\mathfrak{m}_{n-1}(\mathfrak{g})[\widetilde{M}: M]^{(n-2) / 2} \prod_{v \mid \mathfrak{e}} \lambda_{v} \cdot \begin{cases}1 & \text { if } n \text { is even }, \\ N\left(\mathfrak{d}_{K}\right)^{-1 / 2} & \text { if } n \text { is odd }\end{cases}
$$

with the value $\lambda_{v}$ given as follows:

$$
\begin{cases}1 & \text { if } s_{v}=1, v \mid 2, \text { and } \delta q \in \mathfrak{g}_{v}^{\times} F_{v}^{\times 2}, \\ 2^{-1}\left(1+q_{v}^{-(n-2) / 2}\right) & \text { if } s_{v}=1 \text { and } \delta q \in \pi_{v} \mathfrak{g}_{v}^{\times} F_{v}^{\times 2}, \\ 2^{-1}\left(1+q_{v}\right)^{-1}\left(1+q_{v}^{1-\ell}\right)\left(1+q_{v}^{-\ell}\right) & \text { if } s_{v}=2,\left(\mathfrak{d}_{K}\right)_{v}=\mathfrak{r}_{v}, \text { and } \widehat{N_{W_{v}}} \neq N_{W_{v}}, \\ 2^{-1} & \text { if } s_{v}=2 \text { and }\left(\mathfrak{d}_{K}\right)_{v} \neq \mathfrak{r}_{v} \\ 2^{-1}\left(1+q_{v}\right)^{-1}\left(1-q_{v}^{2-n}\right) & \text { if } s_{v}=3 \text { and } \delta q \in \mathfrak{g}_{v}^{\times} F_{v}^{\times 2} \\ 2^{-1}\left(1-q_{v}^{-(n-2) / 2}\right) & \text { if } s_{v}=3 \text { and } \delta q \in \pi_{v} \mathfrak{g}_{v}^{\times} F_{v}^{\times 2} \\ 2^{-1}\left(1+q_{v}\right)^{-1}\left(1-q_{v}^{1-\ell}\right)\left(1-q_{v}^{-\ell}\right) & \text { if } s_{v}=4 .\end{cases}
$$

Here $\widetilde{M}=\{x \in W \mid 2 \psi(x, M) \subset \mathfrak{g}\}$, $\mathfrak{e}$ is the product of all prime ideals for which $\widetilde{M_{v}} \neq M_{v}$, and $\mathfrak{r}$ is the maximal order of $K ; s_{v}$ is the core dimension of $\left(W_{v}, \psi_{v}\right)$, $\widetilde{N_{W_{v}}}=\left\{x \in Z_{W_{v}} \mid 2 \psi_{v}\left(x, N_{W_{v}}\right) \subset \mathfrak{g}_{v}\right\}, N_{W_{v}}$ (resp. $\left.Z_{W_{v}}\right)$ is defined in a similar way to $N_{v}\left(\right.$ resp. $\left.Z_{v}\right)$ with $M_{v}\left(\right.$ resp. $\left.W_{v}\right)$ in place of $L_{v}\left(\right.$ resp. $\left.V_{v}\right)$ in $(2.6)$, and $q_{v}$ is the norm of the prime ideal of $\mathfrak{g}_{v}$.

\section{The index $\left[C(M): S O^{\psi}(W)_{\mathrm{A}} \cap C(L)\right]$.}

3.1. We will determine $\left[C(M): S O^{\psi}(W)_{\mathbf{A}} \cap C(L)\right]$ in $(5)$ in the introduction, with a fixed element $h$ of $L[q, \mathfrak{b}]$ and a maximal lattice $M=L \cap W$ in $W$. We start with the 
equality

$$
\left[C(M): S O^{\psi}(W)_{\mathbf{A}} \cap C(L)\right]=\prod_{v \in \boldsymbol{h}}\left[C\left(M_{v}\right): S O^{\psi_{v}}\left(W_{v}\right) \cap C\left(L_{v}\right)\right]
$$

Here we regard $S O^{\psi_{v}}\left(W_{v}\right)$ as the subgroup $\left\{\alpha \in S O^{\varphi_{v}}\left(V_{v}\right) \mid h \alpha=h\right\}$ of $S O^{\varphi_{v}}\left(V_{v}\right)$ and put $C\left(M_{v}\right)=\left\{\gamma \in S O^{\psi_{v}}\left(W_{v}\right) \mid M_{v} \gamma=M_{v}\right\}$ and $C\left(L_{v}\right)=\left\{\gamma \in S O^{\varphi_{v}}\left(V_{v}\right) \mid L_{v} \gamma=L_{v}\right\}$. It is sufficient to determine the index $\left[C\left(M_{v}\right): S O^{\psi_{v}}\left(W_{v}\right) \cap C\left(L_{v}\right)\right]$ for given $h \in L_{v}\left[q, \mathfrak{b}_{v}\right]$ and $v \in \boldsymbol{h}$. Hereafter until the end of this section we fix $v \in \boldsymbol{h}$ and drop the subscript $v$ for simplicity.

We take a Witt decomposition of $V$ with respect to $\varphi$ as in (2.6) and fix such a decomposition throughout this section. We use the same letter $\varphi$ for the restriction of $\varphi$ to $Z$.

Before proceeding our arguments, we need to change $h$ for a representative for the set $\left\{x \in L \mid \varphi[x]=c^{2} q\right\}$ under the modulo $C(L)$ with some $c \in F^{\times}$, as stated in [10, Theorem 3.5]. Let us here explain the idea of changing $h$. First, we can find $c \in F^{\times}$ satisfying $c^{2} q \in \mathfrak{g}$ and $c \mathfrak{b} \subset 2^{-1} \mathfrak{g}$. Then we easily see that the $\mathfrak{g}$-lattice $L+\mathfrak{g} c h$ contains $L$ and satisfies $\varphi[L+\mathfrak{g} c h] \subset \mathfrak{g}$. From the maximality of $L$, this lattice coincides with $L$, and hence we have $c h \in\left\{x \in L \mid \varphi[x]=c^{2} q\right\}$. [10, Theorem 3.5] gives a complete set of representatives for $\left\{x \in L \mid \varphi[x]=c^{2} q\right\} / C(L)$. Hence there exists some representative $u$ such that $(c h) \gamma=u$ with some $\gamma \in C(L)$. The element $u$ can be taken from the subset $N+\left(\mathfrak{g} e_{r}+\mathfrak{g} f_{r}\right)$ of $V$ in the notation of (2.6). It should be noted that the index $\left[C(M): S O^{\psi}(W) \cap C(L)\right]$ defined with $h$ is the same as the index defined with $c h \gamma$. Also note that $L \cap(F u)^{\perp}$ is a maximal lattice in $(F u)^{\perp}$. Thus we may determine the index $\left[C\left(L \cap(F u)^{\perp}\right): S O^{\varphi}\left((F u)^{\perp}\right) \cap C(L)\right]$ instead of $\left[C(M): S O^{\psi}(W) \cap C(L)\right]$. In this sense, hereafter we identify $h$ with $u$. Then we have

$$
h \in \begin{cases}N & \text { if } N[q] \neq \emptyset, \\ N+\left(\mathfrak{g} e_{r}+\mathfrak{g} f_{r}\right) & \text { if } N[q]=\emptyset,\end{cases}
$$

where $N[q]=\{z \in N \mid \varphi[z]=q\}$. We also note that $h \in L[q, \mathfrak{b}]$ with $q \in \mathfrak{g}$ and $\mathfrak{b} \subset 2^{-1} \mathfrak{g}$, and that

\section{$L \cap W$ is maximal in $W$}

under our identification. According to (3.2) and [10, Theorem 5.3], the ideal $\mathfrak{b}$ satisfies

$$
2 \mathfrak{b}=\mathfrak{p}^{\tau(q)},
$$

where the value $\tau(q)$ only depends on $(V, \varphi)$ and $q$ and is given in [10, Theorem 3.5]. Let $q \mathfrak{g}=\mathfrak{p}^{\nu}$.

For a subspace $X$ of $V$ we regard $O^{\varphi}(X)$ as the subgroup $\left\{\alpha \in O^{\varphi}(V) \mid x \alpha=\right.$ $x$ for any $\left.x \in X^{\perp}\right\}$ of $O^{\varphi}(V)$. In particular, $O^{\psi}(W)=\left\{\alpha \in O^{\varphi}(V) \mid h \alpha=h\right\}$. We understand $O^{\psi}(Y)$ in a way similar for a subspace $Y$ of $W$. Put $D(L)=\left\{\gamma \in O^{\varphi}(V) \mid L \gamma=L\right\}$ and $D(M)=\left\{\gamma \in O^{\psi}(W) \mid M \gamma=M\right\}$. 
Proposition 3.2. Let the notation be the same as in Section 3.1. Then the following assertions hold:

(i) $\left[O^{\psi}(W) \cap D(L): S O^{\psi}(W) \cap C(L)\right]=2$.

(ii) $\left[C(M): S O^{\psi}(W) \cap C(L)\right]=\left[D(M): O^{\psi}(W) \cap D(L)\right]$.

Proof. By [6, Lemma 6.8] we obtain $[D(M): C(M)]=2$. Assertion (ii) follows from this and (i). Let us show (i). Since $\operatorname{det}\left(O^{\psi}(W) \cap D(L)\right) \subset\{ \pm 1\}$, we have $\left[O^{\psi}(W) \cap\right.$ $\left.D(L): S O^{\psi}(W) \cap C(L)\right] \leq 2$. Thus it is enough to show that there exists an element $\sigma$ of $O^{\psi}(W) \cap D(L)$ such that $\operatorname{det}(\sigma)=-1$. By (3.1) recall that $h \in N$ if $N[q] \neq \emptyset$ and that $h \in N+\left(\mathfrak{g} e_{r}+\mathfrak{g} f_{r}\right)$ if $N[q]=\emptyset$. First suppose that $r \geq 1$ if $N[q] \neq \emptyset$, and that $r \geq 2$ if $N[q]=\emptyset$. Then we have $h \in\left(F e_{1}+F f_{1}\right)^{\perp}$. We can show (i) in a similar way to the case $t=1$ in the proof of [6, Proposition $11.12(\mathrm{v})]$. Next suppose $r=0$. Then $V=Z, n=t \geq 3$, and $L=N$. Since $\widetilde{L} \neq L$ by [8, (29.7)], if $\varphi$ does not satisfy (2.4), then [6, Proposition $11.12(\mathrm{v})]$ is applicable to the present case. Here $\widetilde{L}$ is as in Section 2.1. An argument similar to the proof of [6, Proposition 11.12 (v)] shows (i) if $\varphi$ satisfies (2.4), that is, if $t=3$ and $\operatorname{det}(\varphi) \in \pi \mathfrak{g}^{\times} F^{\times 2}$. This proves (i) in the case $r=0$. Now suppose that $r=1$ and $N[q]=\emptyset$. Then $V=Z+\left(F e_{1}+F f_{1}\right)$ and $\operatorname{dim}_{F}(Z) \geq 1$. If $h \in F e_{1}+F f_{1}$, then (i) can be obtained in a similar way to the case $\widetilde{L}=L$ and $t \neq 1$ in the proof of $[\mathbf{6}$, Proposition $11.12(\mathrm{v})]$. Assume $h \notin F e_{1}+F f_{1}$; assume also $\operatorname{dim}_{F}(V)>3$. Let $z$ be an element of $N$ such that $h \in z+\left(\mathfrak{g} e_{1}+\mathfrak{g} f_{1}\right)$. Then $z \neq 0$ and $V=\left[Z \cap(F z)^{\perp}\right]+\left(F z+F e_{1}+F f_{1}\right)$. We can find the desired $\sigma$ in a similar way to the case $h \in F e_{1}+F f_{1}$.

Finally assume $\operatorname{dim}_{F}(V)=3$. Note that $\varphi$ is isotropic on $V$. In this case we employ the setting of the case $t=3$ in Section 2.3. Then $(V, \varphi)=\left(B^{\circ}, d \beta^{\circ}\right)$, where $B^{\circ}=\{x \in$ $\left.M_{2}(F) \mid \operatorname{tr}(x)=0\right\}, \beta^{\circ}[x]=\operatorname{det}(x)$, and $d \in F^{\times}$. We may assume $d \in \mathfrak{g}^{\times} \sqcup \pi \mathfrak{g}^{\times}$. Then we have a Witt decomposition $B^{\circ}=F g+(F e+F f)$ and $L=\mathfrak{g} g+\mathfrak{g} e+\mathfrak{g} f$ with $g=\left[\begin{array}{cc}1 & 0 \\ 0 & -1\end{array}\right]$, $e=\left[\begin{array}{cc}0 & -d^{-1} \\ 0 & 0\end{array}\right]$, and $f=\left[\begin{array}{ll}0 & 0 \\ 1 & 0\end{array}\right]$. Now recall $N[q]=\emptyset$. Then there are two elements $c \in 2^{-1} \mathfrak{g}$ and $a \in \mathfrak{g}$ such that $c g+a e+f$ belongs to $L\left[\pi^{-2 \tau(q)} q, 2^{-1} \mathfrak{g}\right]$ by the same manner as in [7, Section 1.6], where $\tau(q)$ is in (3.3). Note that $\pi^{\tau(q)}\left(L\left[\pi^{-2 \tau(q)} q, 2^{-1} \mathfrak{g}\right]\right)=L[q, \mathfrak{b}]$. Thus, by virtue of [7, Theorem 1.3], $h \gamma=\pi^{\tau(q)}(c g+a e+f)$ with some $\gamma \in C(L)$. For the same reason as explained in Section 3.1, we may identify $h$ with $c g+a e+f$. Put $\omega=-g-2 c d e$, then $\{h, \omega, \omega h\}$ is an orthogonal $F$-basis of $V$ with respect to $\varphi$. Let $\sigma$ be the element of $G L(V)$ defined by $h \sigma=h, \omega \sigma=-\omega$, and $(\omega h) \sigma=\omega h$. Clearly $\sigma \in O^{\psi}(W), \notin S O^{\psi}(W)$. Moreover, in view of $\varphi[h]=d \cdot \operatorname{det}(h)$, observe that $g \sigma, e \sigma$, and $f \sigma$ belong to $L$. Thus $L \sigma \subset L$. Because $\operatorname{det}(\sigma)=-1$, we obtain $L \sigma=L$. This proves (i) in the remaining case, which completes the proof.

We note that even when $L \cap W$ is not maximal in $W$, the above proposition is true. This can be proven by changing $\tau(q)$ for $j \in \mathbf{Z}$ such that $2 \mathfrak{b}=\mathfrak{p}^{j}$ in the proof.

Lemma 3.3. Let the notation be the same as in Section 3.1. Put

$$
U= \begin{cases}Z & \text { if } N[q] \neq \emptyset, \\ Z+\left(F e_{r}+F f_{r}\right) & \text { if } N[q]=\emptyset,\end{cases}
$$


where $N[q]$ is as in (3.1). Then the following assertions hold:

(i) $W=Z_{W}+\sum_{i=1}^{r_{W}}\left(F e_{i}+F f_{i}\right)$, which is a Witt decomposition of $W$ with respect to $\psi$. Here $Z_{W}=U \cap W, r_{W}=r$ if $N[q] \neq \emptyset$, and $r_{W}=r-1$ if $N[q]=\emptyset$. Moreover,

$$
\operatorname{dim}_{F}\left(Z_{W}\right)= \begin{cases}t-1 & \text { if } N[q] \neq \emptyset, \\ t+1 & \text { if } N[q]=\emptyset\end{cases}
$$

(ii) $M=N_{W}+\sum_{i=1}^{r_{W}}\left(\mathfrak{g} e_{i}+\mathfrak{g} f_{i}\right)$ with $N_{W}=(L \cap U) \cap Z_{W}$. Moreover $N_{W}$ is a maximal lattice in $Z_{W}$, which is given by $N_{W}=\left\{x \in Z_{W} \mid \psi[x] \in \mathfrak{g}\right\}$.

Proof. First assume $N[q] \neq \emptyset$. Then $h \in N$ by (3.1). Assertion (i) and the first assertion of (ii) are trivial in view of (2.6). Since $N=\{x \in Z \mid \varphi[x] \in \mathfrak{g}\}$, we have $N_{W}=\left\{x \in Z_{W} \mid \psi[x] \in \mathfrak{g}\right\}$, which is maximal in $Z_{W}$. This proves our lemma in the case $N[q] \neq \emptyset$. Next assume $N[q]=\emptyset$. Since $h \in N+\left(\mathfrak{g} e_{r}+\mathfrak{g} f_{r}\right)$, we have $W=Z_{W}+\sum_{i=1}^{r_{W}}\left(F e_{i}+F f_{i}\right)$. This is a Witt decomposition because the restriction of $\psi$ to $Z_{W}$ is anisotropic. To see that $\psi$ is anisotropic on $Z_{W}$, put $k=q e_{r}+f_{r}$. Since $\varphi[k]=q$, by $\left[\mathbf{6}\right.$, Lemma 1.5 (ii)], there is $\alpha \in S O^{\varphi}(U)$ such that $h \alpha=k$. With this $\alpha$, we may identify $Z_{W}$ with $U \cap(F k)^{\perp}$. Then $Z_{W}=Z+F g$ with $g=q e_{r}-f_{r}$. If $t=0$, then the assertion is clear. Assume $t \geq 1$ and $\varphi[z+a g]=0$ with $z \in Z$ and $a \in F$. If $a \neq 0$, then $\varphi\left[a^{-1} z\right]=q$, which contradicts the assumption $N[q]=\emptyset$. Thus $a=0$, and hence $z=0$ because $\varphi$ is anisotropic on $Z$. This proves the desired fact. It is obvious that $\operatorname{dim}_{F}\left(Z_{W}\right)=t+1$. Thus we obtain (i). From (2.6) and (i) we have $M=N_{W}+\sum_{i=1}^{r_{W}}\left(\mathfrak{g} e_{i}+\mathfrak{g} f_{i}\right)$. By [6, Lemma $\left.6.3(1)\right], N_{W}$ is maximal in $Z_{W}$ and hence $N_{W}=\left\{x \in Z_{W} \mid \psi[x] \in \mathfrak{g}\right\}$.

Lemma 3.4. Let $U$ be as in Lemma 3.3. Take an element $v$ of $L \cap U$ such that $\varphi(h, v) \mathfrak{g}=\mathfrak{b}$. Then

$$
L \cap U=\mathfrak{g} v+N_{W}
$$

with $N_{W}$ in Lemma 3.3. Moreover, put $h=c v+w$ with $c \in \mathfrak{g}$ and $w \in N_{W}$. Then $c \mathfrak{g}=q \mathfrak{b}^{-1}$ and

$$
\begin{aligned}
O^{\psi}(W) \cap D(L) & =\left\{\gamma \in D(M) \mid\left[w+q \mathfrak{b}^{-1} M\right] \gamma=w+q \mathfrak{b}^{-1} M\right\}, \\
O^{\psi}\left(Z_{W}\right) \cap D(L) & =\left\{\gamma \in D\left(N_{W}\right) \mid\left[w+q \mathfrak{b}^{-1} N_{W}\right] \gamma=w+q \mathfrak{b}^{-1} N_{W}\right\} .
\end{aligned}
$$

Proof. We first remark that the element $v$ in the statement exists whenever $\varphi(h, L)=\mathfrak{b}$. In fact, we have $\varphi(h, L \cap U)=\mathfrak{b}$ as $h \in L \cap U$. Hence there is a generator $\varphi(h, v)$ of $\mathfrak{b}$ over $\mathfrak{g}$ with some $v \in L \cap U$, which is the required element. We also remark that $c$ and $w$ are uniquely determined by $h$ and $v$.

Let us prove (3.5). For any $x \in L \cap U$ we have $\varphi(h, x)=\varphi(h, v) a$ with $a \in \mathfrak{g}$. Thus $x-a v \in N_{W}$, which shows (3.5). Let $h=c v+w$ as in the statement. Since $c \neq 0$, we have $v=c^{-1}(h-w)$, and hence $\mathfrak{b}=q(c \mathfrak{g})^{-1}$. To see (3.6) and (3.7), employing (3.5), observe that $L=\mathfrak{g} v+M$. For any $\gamma \in D(M)$ we see that $\gamma \in O^{\psi}(W) \cap D(L)$ if and only if $L \gamma=L$. That is the case if and only if $v \gamma-v \in M$, since $\varphi(h, v \gamma-v)=0$. As 
$v=c^{-1}(h-w)$, we have thus $O^{\psi}(W) \cap D(L)=\left\{\gamma \in D(M) \mid w \gamma-w \in q \mathfrak{b}^{-1} M\right\}$. This proves (3.6). Noticing that $O^{\psi}\left(Z_{W}\right)$ is identified with the subgroup of $O^{\psi}(W)$, we obtain $O^{\psi}\left(Z_{W}\right) \cap D(L)=\left\{\gamma \in D\left(N_{W}\right) \mid w \gamma-w \in q \mathfrak{b}^{-1} M\right\}$. The condition $w \gamma-w \in q \mathfrak{b}^{-1} M$ can be replaced by $w \gamma-w \in q \mathfrak{b}^{-1} N_{W}$, which proves (3.7). This completes the proof.

We recall that $\mathfrak{b}$ satisfies condition (3.3) under the assumption that $L \cap W$ is maximal in $W$. Then the ideal $q^{\mathfrak{b}^{-1}}$ in Lemma 3.4 satisfies

$$
q \mathfrak{b}^{-1} \subset \mathfrak{p}^{[\nu / 2]} .
$$

In fact, we see that $2 \mathfrak{b} \subset \mathfrak{p}^{[\nu / 2]}$. This can be found in [10, (3.9), (3.12), (3.15), (3.18) and (3.20)] with $\tau(q)$ of (3.3). Hence we have $2 \varphi\left(\pi^{-[\nu / 2]} h, L\right) \subset \mathfrak{g}$. This combined with $\varphi\left[\pi^{-[\nu / 2]} h\right] \in \mathfrak{g}$ implies that $\pi^{-[\nu / 2]} h \in L$ by the maximality of $L$. Thus we obtain $q \mathfrak{p}^{-[\nu / 2]} \subset \mathfrak{b}$, which proves (3.8).

Lemma 3.4 is a key result to determine the index $\left[C(M): S O^{\psi}(W) \cap C(L)\right]$. We note that (3.6) is valid even when $L \cap W$ is not maximal in $W$.

Corollary 3.5. If $q \mathfrak{b}^{-1}=\mathfrak{p}^{[\nu / 2]}$, then $\left[C(M): S O^{\psi}(W) \cap C(L)\right]=1$.

Proof. $\quad$ Let $w$ be as in Lemma 3.4. Since $w=h-c v, c \mathfrak{g}=q \mathfrak{b}^{-1}$, and $h \in \pi^{[\nu / 2]} L$ as seen above, we obtain $w \in \mathfrak{p}^{[\nu / 2]} M$. Then our assertion follows immediately from this, Proposition 3.2 (ii) and (3.6). This completes the proof.

Even when $L \cap W$ is not maximal in $W$, Corollary 3.5 is true with $\min (j,[\nu / 2])$ in place of $[\nu / 2]$, where $j$ is the integer such that $2 \mathfrak{b}=\mathfrak{p}^{j}$. In that case we see that $q \mathfrak{b}^{-1} \subset \mathfrak{p}^{\min (j,[\nu / 2])}$

In view of (3.8) and Corollary 3.5, we only have to consider the case $q \mathfrak{b}^{-1} \subset \mathfrak{p}^{[\nu / 2]+1}$. For this, we need one more lemma:

LEMma 3.6. (i) Let $K$ be an unramified quadratic extension of $F$ and $\mathfrak{r}$ its maximal order. Then $1+\mathfrak{p}=N_{K / F}(1+\mathfrak{p r})$.

(ii) Let $B$ be a division quaternion algebra over $F$ with the main involution $\iota$ of $B$, and $\mathfrak{P}$ the prime ideal of the maximal order of $B$. Put $E_{B}=\left\{u \in B \mid u u^{\iota}=1\right\}$. Then $\left[E_{B}: E_{B} \cap(1+\mathfrak{P})\right]=1+N \mathfrak{p}$.

Proof. Clearly we have $1+\mathfrak{p} \supset N_{K / F}(1+\mathfrak{p r})$. Conversely, for any $a \in 1+\mathfrak{p}$ there exists $b \in \mathfrak{r}^{\times}$such that $a=N_{K / F}(b)$. If $b \in 1+\mathfrak{p r}$, then we obtain (i). Assume that $b \notin 1+\mathfrak{p r}$, that is, $b-1 \in \mathfrak{r}^{\times}$. Put $u=-\left(b^{\rho}-1\right)(b-1)^{-1}$, where $\rho$ is the generator of $\operatorname{Gal}(K / F)$. Then $u b-1=(1-a)(b-1)^{-1} \in \mathfrak{p r}$ and $N_{K / F}(u)=1$. Thus $a=N_{K / F}(u b) \in N_{K / F}(1+\mathfrak{p r})$, and hence we obtain $1+\mathfrak{p} \subset N_{K / F}(1+\mathfrak{p r})$, which proves (i). To prove (ii), let $B=J+J \omega$ with an unramified quadratic extension $J$ of $F$ and $\omega \in B$, as stated in the case $t=3$ in Section 2.3. Put $E=\left\{a \in J \mid a a^{\iota}=1\right\}$. We observe that

$$
E \cap\left[E_{B} \cap(1+\mathfrak{P})\right]=E \cap(1+\mathfrak{p r}), \quad E_{B}=\left[E_{B} \cap(1+\mathfrak{P})\right] \cdot E,
$$

and $E_{B} \cap(1+\mathfrak{P})$ is a normal subgroup of $E_{B}$, where $\mathfrak{r}$ is the maximal order of $J$. From 
these, $[E \cap(1+\mathfrak{p r})] \backslash E$ is isomorphic to $\left[E_{B} \cap(1+\mathfrak{P})\right] \backslash E_{B}$, and hence $\left[E_{B}: E_{B} \cap(1+\mathfrak{P})\right]=$ $[E: E \cap(1+\mathfrak{p r})]=1+N \mathfrak{p}$ by $[\mathbf{3}$, Lemma 3.4].

Proposition 3.7. Assume $q \mathfrak{b}^{-1} \subset \mathfrak{p}^{[\nu / 2]+1}$. For $\gamma \in D\left(N_{W}\right)$ extend $\gamma$ to an element $\widetilde{\gamma}$ of $D(M)$ by setting $\widetilde{\gamma}$ to be the identity map on $Z_{W}^{\perp}$. Here $Z_{W}$ and $N_{W}$ are as in Lemma 3.3. Then the map $\gamma \mapsto \widetilde{\gamma}$ gives a bijection of $D\left(N_{W}\right) /\left[O^{\psi}\left(Z_{W}\right) \cap D(L)\right]$ onto $D(M) /\left[O^{\psi}(W) \cap D(L)\right]$.

Proof. First of all, we note that $\operatorname{dim}_{F}\left(Z_{W}\right) \geq 1$. Indeed, if $\operatorname{dim}_{F}\left(Z_{W}\right)=0$, then by (3.4) we have $t=1$ and $N[q] \neq \emptyset$. The latter condition is equivalent to $c q \in F^{\times 2}$ with an element $c$ of $F^{\times}$as in Section 2.3 under the condition $t=1$. Thus by (3.3) and [10, (3.12)] we see that $q \mathfrak{b}^{-1}=\mathfrak{p}^{[\nu / 2]}$, which is impossible under our assumption. Hence we obtain the desired fact.

It is obvious that the map of our proposition is well-defined and injective. Thus we only have to prove that this map is surjective, that is, for any $\gamma \in D(M)$ there exists $\alpha \in D\left(N_{W}\right)$ such that $\widetilde{\alpha}^{-1} \gamma \in O^{\psi}(W) \cap D(L)$. To do this, employing the notation of Lemma 3.4, we put $h=c v+w$ with $c$ and $w$ there. For a given $\gamma \in D(M)$, put

$$
w \gamma^{-1}=w_{\gamma}+g
$$

with $w_{\gamma} \in N_{W}$ and $g \in \sum_{i=1}^{r_{W}}\left(\mathfrak{g} e_{i}+\mathfrak{g} f_{i}\right)=M \cap\left(Z_{W}\right)^{\perp}$. Let $\alpha \in D\left(N_{W}\right)$. Then by (3.6), $\widetilde{\alpha}^{-1} \gamma \in O^{\psi}(W) \cap D(L)$ if and only if $w \alpha^{-1} \gamma-w \in q \mathfrak{b}^{-1} M$. This is the case if and only if $w_{\gamma} \in\left(w+q \mathfrak{b}^{-1} N_{W}\right) \alpha^{-1}$ and $g \in q \mathfrak{b}^{-1}\left[M \cap\left(Z_{W}\right)^{\perp}\right]$. We assert that $g$ always belongs to $q \mathfrak{b}^{-1}\left[M \cap\left(Z_{W}\right)^{\perp}\right]$. To see this, put $g=\sum_{i=1}^{r_{W}}\left(a_{i} e_{i}+b_{i} f_{i}\right)$ with $a_{i}, b_{i} \in \mathfrak{g}$. Employing Lemma 3.4, we have $a_{i}=2 \psi\left(w, f_{i} \gamma\right) \in c \cdot 2 \varphi(v, M) \subset q \mathfrak{b}^{-1}$. Similarly we have $b_{i} \in q \mathfrak{b}^{-1}$, and hence we obtain the required fact. Consequently, our map is surjective if and only if for any $\gamma \in D(M)$,

$$
w_{\gamma} \in\left(w+q \mathfrak{b}^{-1} N_{W}\right) D\left(N_{W}\right) .
$$

Let us consider the subspace $F w+F w_{\gamma}$ of $Z_{W}$. Put

$$
Z_{\gamma}=F w+F w_{\gamma}, \quad N_{\gamma}=N_{W} \cap Z_{\gamma} .
$$

Then $\psi$ is anisotropic on $Z_{\gamma}$ and $N_{\gamma}=\left\{x \in Z_{\gamma} \mid \psi[x] \in \mathfrak{g}\right\}$, which is a unique maximal lattice in $Z_{\gamma}$. Moreover we consider the set

$$
X=\left\{x \in N_{\gamma} \mid \psi[x]-\psi[w] \in\left(q \mathfrak{b}^{-1}\right)^{2}\right\} .
$$

Since $w \gamma^{-1}-w_{\gamma}=g \in q \mathfrak{b}^{-1} M$, we have $w_{\gamma} \in X$. Therefore if we show that

$$
X \subset\left(w+q \mathfrak{b}^{-1} N_{\gamma}\right) D\left(N_{\gamma}\right),
$$

then the surjectivity holds because $D\left(N_{\gamma}\right) \subset D\left(N_{W}\right)$. We shall prove (3.9).

We first note that

$$
\psi[w] \mathfrak{g}=q \mathfrak{g} .
$$


In fact, recall that $h-w=c v$ and $c \mathfrak{g}=q \mathfrak{b}^{-1}$ by Lemma 3.4. From these (3.10) follows under the assumption $q^{-1} \subset \mathfrak{p}^{[\nu / 2]+1}$. In particular, we find $w \neq 0$, and hence $\operatorname{dim}_{F}\left(Z_{\gamma}\right)=1$ or 2 .

Assume $\operatorname{dim}_{F}\left(Z_{\gamma}\right)=1$. As explained in the case $t=1$ in Section 2.3, we may assume $Z_{\gamma}=F, \psi[a]=c a^{2}$ with some $c \in F^{\times}$, and $N_{\gamma}=\mathfrak{p}^{-[\varepsilon / 2]}$. Here $c \mathfrak{g}=\mathfrak{p}^{\varepsilon}$. Clearly $X=$ $\left\{a \in N_{\gamma} \mid a^{2}-w^{2} \in c^{-1}\left(q \mathfrak{b}^{-1}\right)^{2}\right\}$. On the other hand, since $D\left(N_{\gamma}\right)=O^{\psi}\left(Z_{\gamma}\right)=\{ \pm 1\}$, we have $\left(w+q \mathfrak{b}^{-1} N_{\gamma}\right) D\left(N_{\gamma}\right)=\left(w+q \mathfrak{b}^{-1} N_{\gamma}\right) \cup\left(-w+q \mathfrak{b}^{-1} N_{\gamma}\right)$. Now, take $a \in N_{\gamma}$ such that $a \notin\left(w+q \mathfrak{b}^{-1} N_{\gamma}\right) D\left(N_{\gamma}\right)$. Then $(a-w) \mathfrak{g} \supset q \mathfrak{b}^{-1} N_{\gamma} \mathfrak{p}^{-1}$ and $(a+w) \mathfrak{g} \supset q \mathfrak{b}^{-1} N_{\gamma} \mathfrak{p}^{-1}$. Hence $a^{2}-w^{2} \notin c^{-1}\left(q \mathfrak{b}^{-1}\right)^{2}$. This proves (3.9) in the case $\operatorname{dim}_{F}\left(Z_{\gamma}\right)=1$.

Assume $\operatorname{dim}_{F}\left(Z_{\gamma}\right)=2$. Let $K_{\gamma}$ be the even Clifford algebra of the restriction of $\psi$ to $Z_{\gamma}$. Then we can identify $\left(Z_{\gamma}, \psi\right)$ with $\left(K_{\gamma}, c \kappa_{\gamma}\right)$ as in the case $t=2$ in Section 2.3, with the norm form $\kappa_{\gamma}$ on $K_{\gamma}$ and $c \in F^{\times}$. Let $c \mathfrak{g}=\mathfrak{p}^{\varepsilon}$. By (3.10) we have

$$
X=\left\{a \in N_{\gamma} \mid \kappa_{\gamma}[a] \in \kappa_{\gamma}[w]\left(1+q \mathfrak{b}^{-2}\right)\right\}
$$

Suppose that $K_{\gamma}$ is ramified over $F$. Then we may assume $c \in \mathfrak{g}^{\times} F^{\times 2}$, and hence $N_{\gamma}=\mathfrak{q}^{-\varepsilon}$, where $\mathfrak{q}$ is the prime ideal of the maximal order $\mathfrak{r}$ of $K_{\gamma}$. Thus we have $w+q \mathfrak{b}^{-1} N_{\gamma}=w\left(1+\mathfrak{q}^{\nu} \mathfrak{b}^{-1}\right)$. By [6, Lemma 5.6 (vi)] for any $a \in X$ there exists $b \in \mathfrak{q}^{\nu} \mathfrak{b}^{-1}$ such that $\kappa_{\gamma}[a]=\kappa_{\gamma}[w b]$. Hence $a \in w b O^{\psi}\left(Z_{\gamma}\right)$ by [6, Lemma 1.5 (ii)], which proves (3.9) in the present case. Suppose that $K_{\gamma}$ is unramified over $F$. Because $N_{\gamma}=\mathfrak{p}^{-[\varepsilon / 2]} \mathfrak{r}$, we have $w+q \mathfrak{b}^{-1} N_{\gamma}=w\left(1+q \mathfrak{b}^{-1} \mathfrak{p}^{-[\nu / 2]} \mathfrak{r}\right)$. We can show that

$$
q \mathfrak{b}^{-1}=\mathfrak{p}^{\nu-[\nu / 2]},
$$

which will be seen below. From this and $q \mathfrak{b}^{-1} \subset \mathfrak{p}^{[\nu / 2]+1}$ it follows that $q \in \pi \mathfrak{g}^{\times} F^{\times 2}$ and hence $w+q \mathfrak{b}^{-1} N_{\gamma}=w(1+\mathfrak{p r})$. Also we have $X=\left\{a \in N_{\gamma} \mid \kappa_{\gamma}[a] \in \kappa_{\gamma}[w](1+\mathfrak{p})\right\}$. Taking any $a \in X$, we have $\kappa_{\gamma}[a] \in \kappa_{\gamma}[w(1+\mathfrak{p r})]$ by Lemma 3.6 (i). Thus an argument similar to the case where $K_{\gamma}$ is ramified over $F$ proves (3.9).

Therefore our proof is finished if we show (3.11). To do this, because $\mathfrak{b}=2^{-1} \mathfrak{p}^{\tau(q)}$ by (3.3), we only have to prove $\tau(q)=\kappa+[\nu / 2]$ in the present case, where $2 \mathfrak{g}=\mathfrak{p}^{\kappa}$. To check the value $\tau(q)$ by means of [10, Theorem 3.5], we need the data of the core dimension $t$ of $(V, \varphi)$ and $N[q]$ such that

the core subspace $Z_{W}$ of $(W, \psi)$ contains the 2-dimensional subspace $Z_{\gamma}$ whose discriminant field $K_{\gamma}$ is unramified over $F$.

We distinguish the several possible dimensions for the core $Z_{W}$.

First assume $\operatorname{dim}_{F}\left(Z_{W}\right)=2$. Then $Z_{\gamma}=Z_{W}$, and hence $K_{\gamma}$ is the discriminant field of $Z_{W}$. By (3.4) the following two cases may happen: (a) $t=1$; (b) $t=3$. Let us show that case (b) is impossible as $\operatorname{det}(\psi) \in \mathfrak{g}^{\times} F^{\times 2}$. Indeed, suppose $t=3$; then $h \in Z$ and $Z=F h+Z_{\gamma}$. Moreover, $(Z, \varphi)$ is isomorphic to $\left(B^{\circ}, d \beta^{\circ}\right)$ via the map $\zeta$ of $B^{\circ}$ onto $Z$ with the same notations as in the case $t=3$ in Section 2.3; particularly, $B$ is a division quaternion algebra over $F$. Here $d=(-1)^{r} q \operatorname{det}(\psi)$. Put $k=h \zeta^{-1}$ and $J=F+F k$ in $B$. By the same way as in [6, Section 11.2], we have $B=J+J \omega$ and $B^{\circ}=F k+J \omega$ with a suitable $\omega \in B^{\times}$such that $\omega^{2} \notin \kappa_{J}\left[J^{\times}\right]$, where $\kappa_{J}$ is the norm form on $J$. Then $\left(Z_{\gamma}, \psi\right)$ is isomorphic to $\left(J \omega, d \beta^{\circ}\right)$, which is also isomorphic to $\left(J,-d \omega^{2} \kappa_{J}\right)$. 
Therefore $J$ is isomorphic to $K_{\gamma}$, from which we find that $J$ is an unramified quadratic extension of $F$. Thus we may take $\omega$ so that $\omega^{2}=\pi \notin \kappa_{J}\left[J^{\times}\right]=\mathfrak{g}^{\times} F^{\times 2}$. On the other hand, $Z_{\gamma}$ has the element $w(\neq 0)$, so that $\left(Z_{\gamma}, \psi\right)$ is isomorphic to $\left(K_{\gamma}, q \kappa_{\gamma}\right)$ because of (3.10). From these isomorphisms of $\left(Z_{\gamma}, \psi\right)$ we have $-d \pi \mathfrak{g}^{\times} F^{\times 2}=q \mathfrak{g}^{\times} F^{\times 2}$, that is, $\operatorname{det}(\psi) \in \pi \mathfrak{g}^{\times} F^{\times 2}$. Therefore case (b) does not happen. Hence we may assume case (a). Put $Z=F$ and $\varphi[a]=c a^{2}$ on $Z$ with some $c \in F^{\times}$. To check $\tau(q)$, observe that $F(\sqrt{c q})$ is the discriminant field of $K_{W}$, which is unramified over $F$. This together with $[\mathbf{1 0},(3.12)]$ leads $\tau(q)=\kappa+[\nu / 2]$, which proves (3.11). At the same time, we see that $q \mathfrak{b}^{-1} \subset \mathfrak{p}^{[\nu / 2]+1}$ if and only if $t=1$ and $q \in \pi \mathfrak{g}^{\times} F^{\times 2}$ under the assumption that the discriminant field of 2-dimensional $Z_{W}$ is unramified over $F$, which will be used in the proof of Theorem 3.8 below.

Next assume $\operatorname{dim}_{F}\left(Z_{W}\right)=3$. Then $t=2$ or $t=4$. Let $Z_{W}=F z+Z_{\gamma}$ with some $z$ such that $\psi\left(z, Z_{\gamma}\right)=\{0\}$. Then we can show that $\operatorname{det}(\varphi) \in \pi \mathfrak{g}^{\times} F^{\times 2}$ in a similar way to the case $\operatorname{dim}_{F}\left(Z_{W}\right)=2$ and $t=3$. From this the case $t=4$ is impossible as $\operatorname{det}(\varphi) \in \mathfrak{g}^{\times} F^{\times 2}$. Consequently if 3 -dimensional $Z_{W}$ satisfies (3.12), then $t=2$. In this case we have $N[q]=\emptyset$, which is the case if and only if $c^{-1} q \notin \kappa\left[K^{\times}\right]$with $K, \kappa$, and $c$ as in the case $t=2$ in Section 2.3. By [10, (3.15)] this implies $\tau(q)=\kappa+[\nu / 2]$. Hence we obtain (3.11).

Finally assume $\operatorname{dim}_{F}\left(Z_{W}\right)=4$. Then $t=3$ and $N[q]=\emptyset$. We have $(Z, \varphi)=$ $\left(B^{\circ}, d \beta^{\circ}\right)$ with the same notations in the case $t=3$ in Section 2.3. The condition of $N[q]=\emptyset$ means $F(\sqrt{-d q})=F$. Then $[\mathbf{1 0},(3.18)]$ shows $\tau(q)=\kappa+[\nu / 2]$, which proves (3.11). Note that (3.11) holds when $\operatorname{dim}_{F}\left(Z_{W}\right)=4$, without condition (3.12), which will be needed in the proof of Theorem 3.8 below. This completes the proof of our proposition.

Let $Q(\varphi)$ be the characteristic algebra of $(V, \varphi)$, that is, $Q(\varphi)$ is a quaternion algebra over $F$ such that $A(V)$ is isomorphic to $M_{s}(Q(\varphi))$ if $n \in 2 \mathbf{Z}$, or that $A^{+}(V)$ is isomorphic to $M_{s}(Q(\varphi))$ if $n \notin 2 \mathbf{Z}$, with some $0<s \in \mathbf{Z}$ (cf. [8, Section 28]). Note that $Q(\varphi)$ coincides with the characteristic algebra of $(Z, \varphi)$. If $V$ is a vector space of dimension 1 over $F$, then we define $Q(\varphi)$ by $M_{2}(F)$. For a quaternion algebra $B$ over $F$, put $\chi(B)=1$ if $B$ is isomorphic to $M_{2}(F)$, and put $\chi(B)=-1$ if $B$ is a division algebra. For $x \in F$, put

$$
\xi(x)= \begin{cases}1 & \text { if } \sqrt{x} \in F \\ -1 & \text { if } F(\sqrt{x}) \text { is an unramified quadratic extension of } F \\ 0 & \text { if } F(\sqrt{x}) \text { is a ramified quadratic extension of } F\end{cases}
$$

Now we state the main result in this paper.

THEOREm 3.8. Let $V$ be an n-dimensional vector space over a local field $F$ and $\varphi$ a nondegenerate $F$-bilinear symmetric form, where $2<n \in \mathbf{Z}$. Let $L$ be a maximal lattice in $V$ with respect to $\varphi$. Assume $L[q, \mathfrak{b}] \neq \emptyset$ with $q \in F^{\times}$and a fractional ideal $\mathfrak{b}$ of $F$. For an element $h$ of $L[q, \mathfrak{b}]$, put $W=(F h)^{\perp}$ and let $\psi$ be the restriction of $\varphi$ to $W$. We regard $S O^{\psi}(W)$ as the subgroup $\left\{\alpha \in S O^{\varphi}(V) \mid h \alpha=h\right\}$ of $S O^{\varphi}(V)$. Assume that the $\mathfrak{g}$-lattice $L \cap W$ is maximal in $W$. (Then the ideal $\mathfrak{b}$ satisfies the condition of 
(3.3).) Put $M=L \cap W$. Then

$$
\begin{aligned}
& {\left[C(M): S O^{\psi}(W) \cap C(L)\right]} \\
& \quad= \begin{cases}2 & \text { if } t=0 \text { and } q \in \pi \mathfrak{g}^{\times} F^{\times 2} ; \\
& t=1, \delta \in \mathfrak{g}^{\times} F^{\times 2}, \text { and } \xi(\delta q)=0 ; \\
& t=2, \xi(\delta)=-1, \chi(Q(\varphi))=1, \text { and } q \in \pi \mathfrak{g}^{\times} F^{\times 2}, \\
1+N \mathfrak{p} & \text { if } t=1, q \in \pi \mathfrak{g}^{\times} F^{\times 2}, \text { and } \xi(\delta q)=-1 ; \\
& t=2, \xi(\delta)=0, \chi(Q(\psi))=-1, \text { and } \delta q \in \mathfrak{g}^{\times} F^{\times 2} ; \\
& t=3, q \in \pi \mathfrak{g}^{\times} F^{\times 2}, \text { and } \xi(\delta q)=1, \\
1 & \text { otherwise. }\end{cases}
\end{aligned}
$$

Here $t$ is the core dimension of $(V, \varphi), \delta=(-1)^{n(n-1) / 2} \operatorname{det}(\varphi), Q(\varphi)($ resp. $Q(\psi))$ is the characteristic algebra of $(V, \varphi)$ (resp. $(W, \psi))$, and $N \mathfrak{p}$ is the norm of $\mathfrak{p}$.

Proof. We take a Witt decomposition of $V$ with respect to $\varphi$ as in (2.6). As explained in Section 3.1, we retake $h$ as in (3.1) and fix it. Recall that $q \mathfrak{b}^{-1} \subset \mathfrak{p}^{[\nu / 2]}$ by (3.8). If $q \mathfrak{b}^{-1}=\mathfrak{p}^{[\nu / 2]}$, then by Corollary 3.5, $\left[C(M): S O^{\psi}(W) \cap C(L)\right]=1$. Hereafter we suppose that $q \mathfrak{b}^{-1} \subset \mathfrak{p}^{[\nu / 2]+1}$.

By Propositions 3.2 and 3.7, we obtain

$$
\left[C(M): S O^{\psi}(W) \cap C(L)\right]=\left[D\left(N_{W}\right): O^{\psi}\left(Z_{W}\right) \cap D(L)\right]
$$

where $Z_{W}$ and $N_{W}$ are as in Lemma 3.3. Note that $O^{\psi}\left(Z_{W}\right)=D\left(N_{W}\right)$ and $\operatorname{dim}_{F}\left(Z_{W}\right) \geq$ 1. Let us consider the set $\left(w+q \mathfrak{b}^{-1} N_{W}\right) O^{\psi}\left(Z_{W}\right)$ with $w(\neq 0)$ of Lemma 3.4. Then by (3.7) we have

$$
\left(w+q \mathfrak{b}^{-1} N_{W}\right) O^{\psi}\left(Z_{W}\right)=\bigsqcup_{\alpha \in\left[O \psi\left(Z_{W}\right) \cap D(L)\right] \backslash D\left(N_{W}\right)}\left(w \alpha+q \mathfrak{b}^{-1} N_{W}\right) .
$$

Once we obtain the number of representatives of $\left(w+q \mathfrak{b}^{-1} N_{W}\right) O^{\psi}\left(Z_{W}\right)$ under the modulo $q \mathfrak{b}^{-1} N_{W}$, by (3.14) it gives our desired index.

Assume $\operatorname{dim}_{F}\left(Z_{W}\right)=1$. Since $O^{\psi}\left(Z_{W}\right)=\{ \pm 1\}$, we have $\left(w+q \mathfrak{b}^{-1} N_{W}\right) O^{\psi}\left(Z_{W}\right)=$ $\left(w+q \mathfrak{b}^{-1} N_{W}\right) \cup\left(-w+q \mathfrak{b}^{-1} N_{W}\right)$, and hence

$$
\left[D\left(N_{W}\right): O^{\psi}\left(Z_{W}\right) \cap D(L)\right]= \begin{cases}2 & \text { if } 2 w \notin q \mathfrak{b}^{-1} N_{W} \\ 1 & \text { otherwise }\end{cases}
$$

To observe the condition $2 w \notin q \mathfrak{b}^{-1} N_{W}$ more precisely, we may assume $Z_{W}=F$ and $\psi[a]=c_{W} a^{2}$ with $c_{W} \in F^{\times}$. Put $c_{W} \mathfrak{g}=\mathfrak{p}^{\varepsilon}$. Also note that we have $t=0$, or $t=2$ and $c^{-1} q \in \kappa\left[K^{\times}\right]$with $K, c$, and $\kappa$ as in the case $t=2$ in Section 2.3. Then by (3.10) we see that $w \mathfrak{g}=\mathfrak{p}^{(\nu-\varepsilon) / 2}$. We have $N_{W}=\mathfrak{p}^{-[\varepsilon / 2]}$. Combining these with $\mathfrak{b}=2^{-1} \mathfrak{p}^{\tau(q)}$, we see that $2 w \notin q \mathfrak{b}^{-1} N_{W}$ if and only if $\tau(q) \leq \nu-[\nu / 2]-1$. That is the case if and only if $t=0$ and $q \in \pi \mathfrak{g}^{\times} F^{\times 2}$ because the value $\tau(q)$ can be found in [10, (3.9) and (3.15)]. This gives assertion (3.13) in the case $\operatorname{dim}_{F}\left(Z_{W}\right)=1$. 
Assume $\operatorname{dim}_{F}\left(Z_{W}\right)=2$. Then $t=1$ or $t=3$. We identify $\left(Z_{W}, \psi\right)$ with $\left(K_{W}, c_{W} \kappa_{W}\right)$ with $c_{W} \in F^{\times}$, where $K_{W}=A^{+}\left(Z_{W}\right)$ and $\kappa_{W}$ is its norm form. By [6, Section 7.2] we have $O^{\psi}\left(Z_{W}\right)=\{1, \rho\} E$, where $\rho$ is the generator of $\operatorname{Gal}\left(K_{W} / F\right)$ and $E=\left\{a \in K_{W}^{\times} \mid a a^{\rho}=1\right\}$. Then we see that $\left(w+q \mathfrak{b}^{-1} N_{W}\right) O^{\psi}\left(Z_{W}\right)=$ $\bigsqcup_{b \in\left[E \cap\left(1+q \mathfrak{b}^{-1} w^{-1} N_{W}\right)\right] \backslash E}\left(w b+q \mathfrak{b}^{-1} N_{W}\right)$. We put $c_{W} \mathfrak{g}=\mathfrak{p}^{\varepsilon}$. Recall that $N_{W}=\mathfrak{q}^{-\varepsilon}$ if $K_{W}$ is ramified over $F$, and $N_{W}=\mathfrak{p}^{-[\varepsilon / 2]} \mathfrak{r}$ if $K_{W}$ is unramified over $F$. Here $\mathfrak{q}$ is the prime ideal of the maximal order $\mathfrak{r}$ of $K_{W}$. Then by $(3.10), q \mathfrak{b}^{-1} w^{-1} N_{W}=\mathfrak{q}^{\nu} \mathfrak{b}^{-1}$ if $K_{W}$ is ramified over $F$, and $q \mathfrak{b}^{-1} w^{-1} N_{W}=q \mathfrak{b}^{-1} \mathfrak{p}^{-[\nu / 2]} \mathfrak{r}$ if $K_{W}$ is unramified over $F$. Therefore we obtain

$$
\begin{aligned}
{\left[D\left(N_{W}\right): O^{\psi}\left(Z_{W}\right)\right.} & \cap D(L)] \\
& = \begin{cases}{\left[E: E \cap\left(1+\mathfrak{q}^{\nu} \mathfrak{b}^{-1}\right)\right]} & \text { if } K_{W} \text { is ramified over } F, \\
{\left[E: E \cap\left(1+q \mathfrak{b}^{-1} \mathfrak{p}^{-[\nu / 2]} \mathfrak{r}\right)\right]} & \text { if } K_{W} \text { is unramified over } F .\end{cases}
\end{aligned}
$$

Suppose that $K_{W}$ is ramified over $F$. Note that $K_{W}=F(\sqrt{c q})$ if $t=1$, and $K_{W}=$ $F(\sqrt{-d q})$ if $t=3$ with $c$ and $d$ as in Section 2.3. If $t=1$ and $\delta \in \mathfrak{g}^{\times} F^{\times 2}$, checking $\tau(q)$ by $[\mathbf{1 0},(3.12)]$, we have $\mathfrak{q}^{\nu} \mathfrak{b}^{-1}=\mathfrak{d}$, where $\mathfrak{d}$ is the different of $K_{W}$ relative to $F$. Therefore employing [6, Lemma 5.6 (iv) and (iii)], we obtain $\left[E: E \cap\left(1+\mathfrak{q}^{\nu} \mathfrak{b}^{-1}\right)\right]=2$. Similarly for the case where $t=1$ and $\delta \in \pi \mathfrak{g}^{\times} F^{\times 2}$ or $t=3$, we have $\mathfrak{q}^{\nu} \mathfrak{b}^{-1} \supsetneq \mathfrak{d}$ by [10, (3.12) and (3.18)], and then $\left[E: E \cap\left(1+\mathfrak{q}^{\nu} \mathfrak{b}^{-1}\right)\right]=1$ by [6, Lemma 5.6 (iv) and (v)]. Suppose that $K_{W}$ is unramified over $F$. Our assumption $q \mathfrak{b}^{-1} \subset \mathfrak{p}^{[\nu / 2]+1}$ implies that $t=1$ and $q \in \pi \mathfrak{g}^{\times} F^{\times 2}$ as observed in the proof of Proposition 3.7. Note that $\tau(q)=\kappa+[\nu / 2]$. Then applying [3, Lemma 3.4], we obtain $\left[E: E \cap\left(1+q \mathfrak{b}^{-1} \mathfrak{p}^{-[\nu / 2]} \mathfrak{r}\right)\right]=1+N \mathfrak{p}$. This proves (3.13) in the case $\operatorname{dim}_{F}\left(Z_{W}\right)=2$.

Assume $\operatorname{dim}_{F}\left(Z_{W}\right)=3$. Then $t=2$ and $N[q]=\emptyset$, or $t=4$. If $t=4$, then $D(L)$ contains $D(N)=O^{\varphi}(Z)$ with the notation of (2.6). Since $O^{\varphi}(Z)$ contains $O^{\psi}\left(Z_{W}\right)=$ $D\left(N_{W}\right)$, we have $\left[D\left(N_{W}\right): O^{\psi}\left(Z_{W}\right) \cap D(L)\right]=1$.

Suppose $t=2$ and $N[q]=\emptyset$. Recall that the condition $N[q]=\emptyset$ means $c^{-1} q \notin \kappa\left[K^{\times}\right]$ with the notation of the case $t=2$ in Section 2.3. Then $h$ is contained in $L \cap U$, more precisely in this case, it is in $N+\mathfrak{g} e_{r}+\pi^{\tau(q)} f_{r}$ by [10, Theorem 3.5 (iii)]. Since $\varphi\left(h, e_{r}\right) \mathfrak{g}=\mathfrak{b}$, we may take $v$ in Lemma 3.4 as $e_{r}$. Then $\psi[w]=-q$ as $h-w=c v$ with $c$ as in Lemma 3.4. Employing the setting of the case $t=3$ in Section 2.3, we may identify $\left(Z_{W}, \psi\right)$ with $\left(B^{\circ}, \delta q \beta^{\circ}\right)$. Also we have $B=J+J \omega$ and $B^{\circ}=F \eta+J \omega$ with an unramified quadratic extension $J$ of $F, \omega$, and $\eta$ as in Section 2.3. Put $\delta q \mathfrak{g}=\mathfrak{p}^{\varepsilon}$. Then we have $N_{W}=\mathfrak{p}^{-\varepsilon / 2}(\mathfrak{g} \eta+\mathfrak{r} \omega)$ or $N_{W}=\mathfrak{p}^{-(\varepsilon-1) / 2}\left(\mathfrak{g} \eta+\mathfrak{p}^{-1} \mathfrak{r} \omega\right)$ according as $\delta q \in \mathfrak{g}^{\times} F^{\times 2}$ or $\delta q \in \pi \mathfrak{g}^{\times} F^{\times 2}$. Also we see that $N_{W}=B^{\circ} \cap \mathfrak{P}^{-\varepsilon}$, where $\mathfrak{P}$ is the prime ideal of the maximal order $\mathfrak{O}$ of $B$. Then we obtain $O^{\psi}\left(Z_{W}\right)=C \sqcup\left(-1_{Z_{W}}\right) C \sqcup \tau_{\omega} C \sqcup\left(-1_{Z_{W}}\right) \tau_{\omega} C$ with $C=\left\{\tau_{a} \mid a \in \mathfrak{O}^{\times}\right\}$and $\tau_{a}$ defined as in the proof of Lemma 2.4.

Putting $Y=w+q \mathfrak{b}^{-1} N_{W}$ for simplicity, we have $Y O^{\psi}\left(Z_{W}\right)=Y C \cup Y\left(-1_{Z_{W}}\right) C \cup$ $Y \tau_{\omega} C \cup Y\left(-1_{Z_{W}}\right) \tau_{\omega} C$. We shall observe the set $Y C$. Since $Y \tau_{a}=Y$ for every $\tau_{a} \in$ $C \cap\left(O^{\psi}\left(Z_{W}\right) \cap D(L)\right)$ by $(3.7), Y C=\sqcup_{\tau_{a} \in\left[C \cap\left(O^{\psi}\left(Z_{W}\right) \cap D(L)\right)\right] \backslash C}\left(w \tau_{a}+q \mathfrak{b}^{-1} N_{W}\right)$. Let $w=\pi^{-[\varepsilon / 2]}\left(\mu \eta+\pi^{-s} \lambda \omega\right)$ with $\mu \in \mathfrak{g}$ and $\lambda \in \mathfrak{r}$, where $s=0$ or 1 according as $\delta q \in \mathfrak{g}^{\times} F^{\times 2}$ or $\delta q \in \pi \mathfrak{g}^{\times} F^{\times 2}$. Take $\tau_{a} \in C$ with $a=k+\ell \omega$, where $k \in \mathfrak{r}^{\times}$and $\ell \in \mathfrak{r}$. Then we see that 


$$
w \tau_{a}=w+a^{-1} \pi^{-[\varepsilon / 2]}\left(2 \mu \ell \eta \omega+\pi^{-s}\left[\left(k^{\iota}-k\right) \lambda \omega+\left(\ell^{\iota} \lambda-\ell \lambda^{\iota}\right) \omega^{2}\right]\right) .
$$

Hence $\tau_{a} \in O^{\psi}\left(Z_{W}\right) \cap D(L)$ if and only if

$$
2 \mu \ell \eta \omega+\pi^{-s}\left[\left(k^{\iota}-k\right) \lambda \omega+\left(\ell^{\iota} \lambda-\ell \lambda^{\iota}\right) \omega^{2}\right] \in q \mathfrak{b}^{-1} \mathfrak{P}^{-s} .
$$

Assume that $F(\sqrt{\delta})$ is unramified over $F$. Also assume that $\delta q \in \pi \mathfrak{g}^{\times} F^{\times 2}$. Then $q \in \pi \mathfrak{g}^{\times} F^{\times 2}$. Since $N[q]=\emptyset$, which means $c^{-1} q \notin \kappa\left[K^{\times}\right],[\mathbf{1 0},(3.15)]$ shows $q^{\mathfrak{b}^{-1}}=$ $2 q \mathfrak{p}^{-(\nu-1) / 2}$. We may take $J=F+F w$ because $w^{2}=\delta^{-1}$. Then we have $\lambda=0$. Thus by $\delta \beta^{\circ}[w]=-1$, we obtain $\mu \mathfrak{g}=\mathfrak{p}^{(\nu-1) / 2}$. These lead (3.15) for any $\tau_{a} \in C$, that is, $Y C=Y$. From this it follows that $Y\left(-1_{Z_{W}}\right) C=Y \tau_{\omega} C=\left(-w+q \mathfrak{b}^{-1} N_{W}\right) \neq Y$, since $w\left(-1_{Z_{W}}\right)=w \tau_{\omega}=-w$. Also we obtain $Y\left(-1_{Z_{W}}\right) \tau_{\omega} C=Y$. These together with (3.14) prove $\left[D\left(N_{W}\right): O^{\psi}\left(Z_{W}\right) \cap D(L)\right]=2$ under the assumption that $\xi(\delta)=-1$, $N[q]=\emptyset$, and $q \in \pi \mathfrak{g}^{\times} F^{\times 2}$. In particular, the condition $N[q]=\emptyset$ can be replaced by $A(Z) \simeq M_{2}(F)$. That is the case if and only if $\chi(Q(\varphi))=1$, because $Q(\varphi)$ coincides with the characteristic algebra of $(Z, \varphi)$. Similarly we obtain $\left[D\left(N_{W}\right): O^{\psi}\left(Z_{W}\right) \cap D(L)\right]=1$ when $\delta q \in \mathfrak{g}^{\times} F^{\times 2}$. This proves (3.13) in the case where $t=2, \xi(\delta)=-1$, and $N[q]=\emptyset$.

Next assume that $F(\sqrt{\delta})$ is ramified over $F$ and $\delta \in \pi \mathfrak{g}^{\times} F^{\times 2}$. By [10, (3.15)] we find $q \mathfrak{b}^{-1}=q \mathfrak{p}^{-[\nu / 2]}$. Thus we have $q \in \pi \mathfrak{g}^{\times} F^{\times 2}$ as $q \mathfrak{b}^{-1} \subset \mathfrak{p}^{[\nu / 2]+1}$. Observing $\delta \beta^{\circ}[w]=-1$, we have $\lambda \mathfrak{r}=\mathfrak{p}^{(\nu-1) / 2} \mathfrak{r}$ and $\mu \mathfrak{g} \subset \mathfrak{p}^{(\nu+1) / 2}$. Hence (3.15) holds if and only if $k^{\iota}-k \in \mathfrak{p r}$, which is the case if and only if $k \in \mathfrak{g}^{\times}(1+\mathfrak{p r})$ by [3, Lemma 3.4]. Therefore we have $C \cap\left(O^{\psi}\left(Z_{W}\right) \cap D(L)\right)=\left\{\tau_{a} \mid a \in \mathfrak{g}^{\times}(1+\mathfrak{P})\right\}$. Then $\left[C: C \cap\left(O^{\psi}\left(Z_{W}\right) \cap D(L)\right)\right]=$ $\left[\mathfrak{O}^{\times}: 1+\mathfrak{P}\right]\left[\mathfrak{g}^{\times}: 1+\mathfrak{p}\right]^{-1}=1+N \mathfrak{p}$. This finishes the case of $Y C$. To see $Y\left(-1_{Z_{W}}\right) C$, observe that $\left(-1_{Z_{W}}\right) \tau_{b} \in O^{\psi}\left(Z_{W}\right) \cap D(L)$ for any fixed element $b$ of $\mathfrak{r}^{\times} \cap B^{\circ}$. Then we have $Y\left(-1_{Z_{W}}\right) C=Y\left(-1_{Z_{W}}\right) \tau_{b} C=Y C$. Also we have $Y \tau_{\omega} C=Y\left(-1_{Z_{W}}\right) \tau_{\omega} C=Y C$. Consequently $\left[D\left(N_{W}\right): O^{\psi}\left(Z_{W}\right) \cap D(L)\right]=1+N \mathfrak{p}$ if $t=2, \xi(\delta)=0, N[q]=\emptyset$, $\delta \in \pi \mathfrak{g}^{\times} F^{\times 2}$, and $q \in \pi \mathfrak{g}^{\times} F^{\times 2}$. Since $Q(\psi)$ is the division algebra $B$, we see that $N[q]=\emptyset$ if and only if $\chi(Q(\psi))=-1$. This proves (3.13) in the present case.

Finally assume that $F(\sqrt{\delta})$ is ramified over $F$ and $\delta \in \mathfrak{g}^{\times} F^{\times 2}$. Note that $2 \in \mathfrak{p}$. Suppose $\delta q \in \mathfrak{g}^{\times} F^{\times 2}$. Then $q \in \mathfrak{g}^{\times} F^{\times 2}$. Thus by [10, (3.15)] we obtain $q \mathfrak{b}^{-1}=2 D_{F(\sqrt{\delta}) / F}^{-1 / 2} \mathfrak{p}^{2^{-1} \nu+1}$. Here $D_{F(\sqrt{\delta}) / F}$ is the square of an integral ideal of $\mathfrak{g}$ by $[\mathbf{1 0}$, Lemma $5.1(3)]$. From $\delta \beta^{\circ}[w]=-1$ it can be seen that

$$
\pi^{-\varepsilon} \delta\left(\mu^{2} \eta^{2}+\omega^{2} \beta[\lambda]\right)=1 .
$$

Hence we find $\mu \mathfrak{g}=\mathfrak{p}^{\nu / 2}$. We assert that $\lambda \mathfrak{r}=q \mathfrak{b}^{-1} \mathfrak{p}^{-1} \mathfrak{r}$. Indeed, we have $2 \psi\left(w, N_{W}\right) \subset$ $q \mathfrak{b}^{-1}$, since $w=h-c v$. Combining this with $2 \psi\left(w, N_{W}\right)=\delta q \pi^{-\varepsilon}\left(2 \mu \mathfrak{g}+\mathfrak{p} \operatorname{Tr}_{J / F}(\lambda \mathfrak{r})\right)$, we have $\lambda \mathfrak{r} \subset q \mathfrak{b}^{-1} \mathfrak{p}^{-1} \mathfrak{r}$. If $\lambda \mathfrak{r} \neq q \mathfrak{b}^{-1} \mathfrak{p}^{-1} \mathfrak{r}$, then $\delta^{-1} \pi^{\varepsilon}-\mu^{2} \eta^{2}=\omega^{2} \beta[\lambda] \in\left(q \mathfrak{b}^{-1}\right)^{2}$ by (3.16). Thus we have $\delta^{-1} F^{\times 2} \subset \eta^{2}\left(1+q^{-1}\left(q \mathfrak{b}^{-1}\right)^{2}\right) F^{\times 2}$, which is impossible because

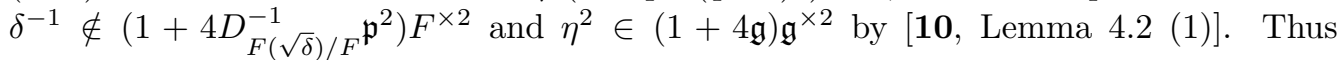
$\lambda \mathfrak{r}=q \mathfrak{b}^{-1} \mathfrak{p}^{-1} \mathfrak{r}$ as desired. In a similar way to the case where $F(\sqrt{\delta})$ is ramified over $F$ and $\delta \in \pi \mathfrak{g}^{\times} F^{\times 2}$, we see that $\left[C: C \cap\left(O^{\psi}\left(Z_{W}\right) \cap D(L)\right)\right]=1+N \mathfrak{p}$. To see $Y \tau_{\omega} C$, let $\lambda \mathfrak{r}=\mathfrak{p}^{m} \mathfrak{r}$ and put $\lambda_{0}=\lambda \pi^{-m}$. Observe that $\tau_{\omega \lambda_{0}^{-1}} \in O^{\psi}\left(Z_{W}\right) \cap D(L)$. Then $Y \tau_{\omega} C=$ $Y \tau_{\omega} \tau_{\lambda_{0}}^{-1} C=Y C$. Also we have $Y\left(-1_{Z_{W}}\right) C=Y\left(-1_{Z_{W}}\right) \tau_{\omega} C=Y C$. Consequently we obtain $\left[D\left(N_{W}\right): O^{\psi}\left(Z_{W}\right) \cap D(L)\right]=1+N \mathfrak{p}$ if $\xi(\delta)=0, \delta \in \mathfrak{g}^{\times} F^{\times 2}, \chi(Q(\psi))=-1$, 
and $q \in \mathfrak{g}^{\times} F^{\times 2}$. When $\delta q \in \pi \mathfrak{g}^{\times} F^{\times 2}$, we see that $\left[D\left(N_{W}\right): O^{\psi}\left(Z_{W}\right) \cap D(L)\right]=1$ in a similar way. This proves (3.13) in the case $\operatorname{dim}_{F}\left(Z_{W}\right)=3$.

Assume $\operatorname{dim}_{F}\left(Z_{W}\right)=4$, that is, $t=3$ and $N[q]=\emptyset$. Then $q \mathfrak{b}^{-1}=\mathfrak{p}^{\nu-[\nu / 2]}$ as shown in the proof of Proposition 3.7. This combined with $q \mathfrak{b}^{-1} \subset \mathfrak{p}^{[\nu / 2]+1}$ implies that $q \in \pi \mathfrak{g}^{\times} F^{\times 2}$, and hence $q \mathfrak{b}^{-1}=(q \mathfrak{p})^{1 / 2}$. We put $\left(Z_{W}, \psi\right)=(B, \beta)$ with the notations as in the case $t=4$ in Section 2.3. Then $N_{W}$ is the maximal order of $B$. We see that $w+q \mathfrak{b}^{-1} N_{W}=w(1+\mathfrak{P})$ because $\psi[w] \mathfrak{g}=q \mathfrak{g}$, where $\mathfrak{P}$ is the prime ideal of $N_{W}$. Since $\beta\left[w(1+\mathfrak{P}) O^{\psi}\left(Z_{W}\right)\right]=\beta[w(1+\mathfrak{P})]$, we have $w(1+\mathfrak{P}) O^{\psi}\left(Z_{W}\right)=w(1+\mathfrak{P}) E_{B}$ with $E_{B}=$ $\{u \in B \mid \beta[u]=1\}$. From these we obtain $w(1+\mathfrak{P}) O^{\psi}\left(Z_{W}\right)=\bigsqcup_{u \in\left[E_{B} \cap(1+\mathfrak{P})\right] \backslash E_{B}} w(1+$ $\mathfrak{P}) u$. Hence by Lemma 3.6 (ii), $\left[D\left(N_{W}\right): O^{\psi}\left(Z_{W}\right) \cap D(L)\right]=1+N \mathfrak{p}$. The condition $N[q]=\emptyset$ is equivalent to $\xi(\delta q)=1$. This proves $(3.13)$ in the case $\operatorname{dim}_{F}\left(Z_{W}\right)=4$. This completes the proof of the theorem.

\section{Applications.}

4.1. Let $(V, \varphi)$ be a quadratic space over $\mathbf{Q}$ such that $\varphi$ is positive definite. Let $L$ be a maximal lattice in $V$. We can compute $\sum_{y \in Y}\left[\Gamma\left(L y^{-1}\right): 1\right]^{-1} \#\left(L y^{-1}\right)[q, \mathfrak{b}]$ in $(2)$ in the introduction under condition (3), using the explicit formula obtained by our result. As mentioned in the introduction, the result is related to the weighted average of the number of primitive representations of an integer by a quadratic form in a specified genus in terms of matrices. For the definition of the genus and class of a symmetric matrix, see $[8$, Section 30.10].

Let $\varphi_{0}$ be the matrix representing $\varphi$ with respect to a $\mathbf{Z}$-basis of $L$. This matrix $\varphi_{0}$ is reduced as a quadratic form in the sense that if $p^{-1} \varphi_{0} \cdot{ }^{t} p^{-1}$ is semi-integral with $p \in M_{n}(\mathbf{Z}) \cap G L_{n}(\mathbf{Q})$, then $\operatorname{det}(p)= \pm 1$ (cf. [8, Section 31.3]). Then the genus of $\varphi_{0}$ consists of all symmetric matrices that are reduced. We identify $(V, \varphi)$ and $L$ with $\left(\mathbf{Q}_{n}^{1}, \varphi_{0}\right)$ and $\mathbf{Z}_{n}^{1}$. Let $\left\{L_{j}\right\}_{j \in J}$ be a complete set of representatives for the $O^{\varphi}(V)$ classes in the genus of all maximal lattices in $V$. Let $L_{j}=\mathbf{Z}_{n}^{1} \alpha_{j}$ with $\alpha_{j} \in G L_{n}(\mathbf{Q})$ for $j \in J$. Then the set of all $O^{\varphi}(V)$-classes in the genus of maximal lattices in $V$ is mapped bijectively onto the set of all $O$-classes in the genus of matrices that are reduced under the correspondence $L_{j} \mapsto \alpha_{j} \varphi_{0} \cdot{ }^{t} \alpha_{j}$. To reformulate the sets $L_{j}[q, \mathfrak{b}]$ for $j \in J$ in this situation, put $\mathfrak{b}=b \mathbf{Z}$. We observe that $\varphi\left(x, L_{j}\right)=\mathfrak{b}$ if and only if $b^{-1} x \varphi_{0} \cdot{ }^{t} \alpha_{j} \mathbf{Z}_{1}^{n}=\mathbf{Z}$ for $x \in V$. Moreover take the smallest positive integer $\ell(\varphi)$ such that $\ell(\varphi)\left(2 \varphi_{0}\right)^{-1}$ is even-integral. Then assigning $b^{-1} x \varphi_{0} \cdot{ }^{t} \alpha_{j}$ to $x$, we get a bijection of $L_{j}[q, \mathfrak{b}]$ onto the set

$$
\left\{y \in \mathbf{Z}_{n}^{1} \mid y \widetilde{\varphi_{j}} \cdot{ }^{t} y=\widetilde{q}, y \mathbf{Z}_{1}^{n}=\mathbf{Z}\right\}
$$

where $\widetilde{\varphi_{j}}=\ell(\varphi)\left(2 \alpha_{j} \varphi_{0} \cdot{ }^{t} \alpha_{j}\right)^{-1}$ and $\widetilde{q}=2^{-1} \ell(\varphi) q b^{-2}$. Thus $\# L_{j}[q, \mathfrak{b}]$ coincides with $\#\left\{y \in \mathbf{Z}_{n}^{1} \mid y \widetilde{\varphi_{j}} \cdot{ }^{t} y=\widetilde{q}, y \mathbf{Z}_{1}^{n}=\mathbf{Z}\right\}$, which is the number of primitive solutions of the equation $\widetilde{\varphi_{j}}[y]=\widetilde{q}$. We denote the latter number by $B\left(\widetilde{\varphi_{j}}, \widetilde{q}\right)$. Note that $\widetilde{q}$ is an evenpositive integer and depends on $\mathfrak{b}$. We also note that there is a relation between the number $B(\widetilde{\varphi}, \widetilde{q})$ of primitive solutions and the number $A(\widetilde{\varphi}, \widetilde{q})$ of solutions of $\widetilde{\varphi}[x]=\widetilde{q}$ in $\mathbf{Z}_{n}^{1}$ as follows: 


$$
B(\widetilde{\varphi}, \widetilde{q})=\sum_{\substack{d \\ d^{2} \mid \widetilde{q}}} \mu(d) A\left(\widetilde{\varphi}, d^{-2} \widetilde{q}\right)
$$

where $\mu$ is the Möbius function and $d$ runs over all positive integer such that $d^{2} \mid \widetilde{q}$.

The correspondence $\alpha_{j} \varphi_{0} \cdot{ }^{t} \alpha_{j} \mapsto \widetilde{\varphi_{j}}$ gives a bijection of the set of all $O$-classes in the genus of $\varphi_{0}$ onto the set of all $O$-classes in the genus of $\widetilde{\varphi}$ with $\widetilde{\varphi}=\ell(\varphi)\left(2 \varphi_{0}\right)^{-1}$. Put $\Gamma^{\cdot}\left(L_{j}\right)=\left\{\gamma \in O^{\varphi}(V) \mid L_{j} \gamma=L_{j}\right\}$ and $E\left(\widetilde{\varphi_{j}}\right)=\#\left\{\left.\alpha \in G L_{n}(\mathbf{Z})\right|^{t} \alpha \widetilde{\varphi_{j}} \alpha=\widetilde{\varphi_{j}}\right\}$. Then $\left[\Gamma \cdot\left(L_{j}\right): 1\right]=E\left(\widetilde{\varphi_{j}}\right)$ via the isomorphism $\gamma \mapsto \alpha_{j} \gamma \alpha_{j}^{-1}$. Put $M(\widetilde{\varphi})=\sum_{j \in J} E\left(\widetilde{\varphi_{j}}\right)^{-1}$ as was defined in $[\mathbf{9},(41)]$ due to Siegel.

Now we have $\mathfrak{m}\left(S O^{\varphi}(V), C(L)\right)=2 \mathfrak{m}\left(O^{\varphi}(V), D(L)\right)$ by [3, Lemma 5.6 (1)], where $C(L)$ is of (1) in the introduction and $D(L)=\left\{\gamma \in O^{\varphi}(V)_{\mathbf{A}} \mid L \gamma=L\right\}$. Also we see that $\sum_{y \in Y}\left[\Gamma\left(L y^{-1}\right): 1\right]^{-1} \#\left(L y^{-1}\right)[q, \mathfrak{b}]=2 \sum_{j \in J}\left[\Gamma^{\cdot}\left(L_{j}\right): 1\right]^{-1} \# L_{j}[q, \mathfrak{b}]$ in a similar way to the proof of [3, Lemma $5.6(1)]$. Consequently we obtain

$$
\frac{\sum_{y \in Y}\left[\Gamma\left(L y^{-1}\right): 1\right]^{-1} \#\left(L y^{-1}\right)[q, \mathfrak{b}]}{\mathfrak{m}\left(S O^{\varphi}(V), C(L)\right)}=\frac{\sum_{j \in J} E\left(\widetilde{\varphi_{j}}\right)^{-1} B\left(\widetilde{\varphi_{j}}, \widetilde{q}\right)}{M(\widetilde{\varphi})} .
$$

Via equality (4.1), we can derive a result on primitive solutions from our result concerning $L[q, \mathfrak{b}]$. For example, let $V=\mathbf{Q}_{6}^{1}$ and $\varphi[x]=\sum_{i=1}^{6} x_{i}^{2}$ for $x=\left(x_{i}\right)_{i=1}^{6} \in V$. Also put $L=\mathbf{Z} e_{1}+\mathbf{Z} e_{2}+\sum_{i=2}^{3}\left(\mathbf{Z} e_{2 i-1}+\mathbf{Z} f_{2 i}\right)$ with the standard basis $\left\{e_{i}\right\}_{i=1}^{6}$ of $V$ and $f_{2 i}=2^{-1}\left(e_{2 i-3}+e_{2 i-2}+e_{2 i-1}+e_{2 i}\right)$. Then [2, Section 3.2] shows that $L$ is a maximal lattice in $V$. The genus of all maximal lattices in $V$ coincides with the $O^{\varphi}(V)$ class of $L$, and also with the $S O^{\varphi}(V)$-class of $L$, which is explained in [6, Section 12.12]. By applying our result to the present case, we can compute $\# L[q, \mathbf{Z}]$ for a squarefree positive integer $q$; see (4.4) below. Now the above $\varphi_{0}$ in this case is given by $\varphi_{0}=\alpha \cdot{ }^{t} \alpha$ with $\alpha \in G L_{6}(\mathbf{Q})$ such that ${ }^{t} \alpha=\left[{ }^{t} e_{1}{ }^{t} e_{2}{ }^{t} e_{3}{ }^{t} f_{4}{ }^{t} e_{5}{ }^{t} f_{6}\right]$. Looking at $\left(2 \varphi_{0}\right)^{-1}$, we find $\ell(\varphi)=4$. This can also be seen by [8, Section 31.5 and (29.9)]. Thus applying (4.1) with $\mathfrak{b}=\mathbf{Z}$, we have $\# L[q, \mathbf{Z}]=B(\widetilde{\varphi}, \widetilde{q})$, where $\widetilde{\varphi}=4\left(2 \varphi_{0}\right)^{-1}$ and $\widetilde{q}=2 q$. Therefore $\# L[q, \mathbf{Z}]$ equals the number of primitive solutions of

$$
\begin{aligned}
6 x_{1}^{2} & +6 x_{2}^{2}+4 x_{3}^{2}+16 x_{4}^{2}+4 x_{5}^{2}+8 x_{6}^{2}+2\left(4 x_{1} x_{2}+2 x_{1} x_{3}-8 x_{1} x_{4}-2 x_{1} x_{5}\right. \\
& \left.+4 x_{1} x_{6}+2 x_{2} x_{3}-8 x_{2} x_{4}-2 x_{2} x_{5}+4 x_{2} x_{6}-4 x_{3} x_{4}+4 x_{4} x_{5}-8 x_{4} x_{6}-4 x_{5} x_{6}\right)=2 q .
\end{aligned}
$$

4.2. Let $V=\mathbf{Q}_{n}^{1}$ and $\varphi[x]=\sum_{i=1}^{n} x_{i}^{2}$ with $n=6,8$, or 10 , where $x=\left(x_{i}\right)_{i=1}^{n} \in V$. Take a maximal lattice $L$ in $V$. As applications of our result, for a given squarefree positive integer $q$ we give an explicit formula for $\# L[q, \mathfrak{b}]$ or $\sum_{y \in Y}\left[\Gamma\left(L y^{-1}\right)\right.$ : $1]^{-1} \#\left(L y^{-1}\right)[q, \mathfrak{b}]$ according as $n=6$ or 8 , or $n=10$, with $\mathfrak{b}$ satisfying condition (3); see Proposition 4.3 below. To state the formula, we mention some basic facts.

Take $h \in L[q]=\{x \in L \mid \varphi[x]=q\}$. Put $W=(\mathbf{Q} h)^{\perp}$ and let $\psi$ be the restriction of $\varphi$ to $W$. Then by $[\mathbf{1 0}$, Theorem 6.3$]$,

$$
L \cap W \text { is maximal in } W \text { if and only if } h \in L[q, \mathfrak{b}] .
$$

Here the ideal $\mathfrak{b}$ is given by 


$$
\mathfrak{b}=2^{-1} \prod_{p}(p \mathbf{Z})^{\tau_{p}(q)}
$$

with $\tau_{p}(q)$ in $[\mathbf{1 0}$, Theorem 3.5] depending only on $(V, \varphi)$ and $q$. Here $p$ runs over all prime numbers. By $[\mathbf{6},(12.17)]$ we have $L[q]=L\left[q, 2^{-1} \mathbf{Z}\right] \sqcup L[q, \mathbf{Z}]$, since $q$ is squarefree. Thus $\mathfrak{b}$ equals $2^{-1} \mathbf{Z}$ or $\mathbf{Z}$. More precisely, [10, (7.1)] shows that

$$
\mathfrak{b}= \begin{cases}\mathbf{Z} & \text { if } n=6 \text { and } q \equiv 2,3 \quad(\bmod 4), \\ 2^{-1} \mathbf{Z} & \text { if } n=6 \text { and } q \equiv 1 \quad(\bmod 4), \\ 2^{-1} \mathbf{Z} & \text { if } n=8, \\ \mathbf{Z} & \text { if } n=10 \text { and } q \equiv 1,2 \quad(\bmod 4), \\ 2^{-1} \mathbf{Z} & \text { if } n=10 \text { and } q \equiv 3 \quad(\bmod 4) .\end{cases}
$$

If $n=6$ or 8 , then we see that $\#\left(S O^{\varphi}(V) \backslash S O^{\varphi}(V)_{\mathbf{A}} / C(L)\right)=1$ from [6, Section 12.12]. Assume $n=10$. From [2, Section 3.2] and [6, Lemma 9.23 (i)], \# $\left(S O^{\varphi}(V) \backslash S O^{\varphi}(V)_{\mathbf{A}} / C(L)\right)$ equals 2 and $\left\{L_{1}, L_{2}\right\}$ gives a complete set of representatives for the $S O^{\varphi}(V)$-classes in the genus of all maximal lattices in $V$. Here

$$
\begin{gathered}
L_{1}=N_{1}+N_{2}, \quad L_{2}=\mathbf{Z} e_{1}+\mathbf{Z} e_{2}+\sum_{i=2}^{5}\left(\mathbf{Z} e_{2 i-1}+\mathbf{Z} f_{2 i}\right) \\
N_{1}=\mathbf{Z} e_{1}+\mathbf{Z} e_{2}+\sum_{i=2}^{3}\left(\mathbf{Z} e_{2 i-1}+\mathbf{Z} f_{2 i}\right)+\mathbf{Z} g_{7}+\mathbf{Z} f_{8}, \quad N_{2}=\mathbf{Z} e_{9}+\mathbf{Z} e_{10}
\end{gathered}
$$

with the standard basis $\left\{e_{i}\right\}_{i=1}^{10}$ of $V, f_{2 i}=2^{-1}\left(e_{2 i-3}+e_{2 i-2}+e_{2 i-1}+e_{2 i}\right)$, and $g_{7}=$ $2^{-1}\left(e_{1}+e_{3}+e_{5}+e_{7}\right)$. Note that $N_{1}$ (resp. $\left.N_{2}\right)$ is a maximal lattice in $\sum_{i=1}^{8} \mathbf{Q} e_{i}$ (resp. $\left.\mathbf{Q} e_{9}+\mathbf{Q} e_{10}\right)$ by $[\mathbf{2}$, Lemma 3.1].

Proposition 4.3. Let $V=\mathbf{Q}_{n}^{1}$ and $\varphi[x]=\sum_{i=1}^{n} x_{i}^{2}$ for $x=\left(x_{i}\right)_{i=1}^{n} \in V$, with $n=6,8$, or 10 . Let $L$ be a maximal lattice in $V$. Then for a squarefree positive integer $q$ the following assertions hold:

(i) Assume $n=6$. Then we have

$$
\begin{aligned}
& \# L[q, \mathbf{Z}]=\prod_{p \mid q}\left(p^{2}+\left(\frac{-1}{p}\right)\right) \cdot\left\{\begin{array}{lll}
3 & \text { if } q \equiv 2 \quad(\bmod 8) \\
5 & \text { if } q \equiv 6 \quad(\bmod 8) \\
2^{3} & \text { if } q \equiv 3 \quad(\bmod 4)
\end{array}\right. \\
& \# L\left[q, 2^{-1} \mathbf{Z}\right]=60 \prod_{p \mid q}\left(p^{2}+\left(\frac{-1}{p}\right)\right) \text { if } q \equiv 1 \quad(\bmod 4) .
\end{aligned}
$$

Here $p$ runs over all prime factors of $q$ and $\left(\frac{-1}{p}\right)$ is the quadratic residue symbol. We understand that $\prod_{p \mid q}=1$ if $q=1$.

(ii) Assume $n=8$. Then we have 


$$
\# L\left[q, 2^{-1} \mathbf{Z}\right]=240 \prod_{p \mid q}\left(p^{3}+1\right)
$$

(iii) Assume $n=10$. When $q \equiv 1,2(\bmod 4)$, we have

$$
\frac{\# L_{1}[q, \mathbf{Z}]}{2^{16} \cdot 3^{5} \cdot 5^{2} \cdot 7}+\frac{\# L_{2}[q, \mathbf{Z}]}{2^{17} \cdot 3^{4} \cdot 5^{2} \cdot 7}=\frac{1}{2^{19} \cdot 3^{5} \cdot 5^{2} \cdot 7} c(q) \prod_{p \mid q}\left(p^{4}+\left(\frac{-1}{p}\right)\right),
$$

where $L_{1}$ and $L_{2}$ are given in (4.3) and

$$
c(q)=\left\{\begin{array}{lll}
2^{5} & \text { if } q \equiv 1 \quad(\bmod 4) \\
17 & \text { if } q \equiv 2 \quad(\bmod 8) \\
3 \cdot 5 & \text { if } q \equiv 6 \quad(\bmod 8)
\end{array}\right.
$$

When $q \equiv 3(\bmod 4)$, we have

$$
\frac{\# L_{1}\left[q, 2^{-1} \mathbf{Z}\right]}{2^{16} \cdot 3^{5} \cdot 5^{2} \cdot 7}+\frac{\# L_{2}\left[q, 2^{-1} \mathbf{Z}\right]}{2^{17} \cdot 3^{4} \cdot 5^{2} \cdot 7}=\frac{17}{2^{15} \cdot 3^{4} \cdot 5 \cdot 7} \prod_{p \mid q}\left(p^{4}+\left(\frac{-1}{p}\right)\right) .
$$

Proof. Assume $n=6$. Let us compute \#L[q,Z] when $q \equiv 2,3(\bmod 4)$. Then $[\mathbf{1 0}$, Theorem 7.5] asserts that $L[q, \mathbf{Z}] \neq \emptyset$. Thus we can take an element $h$ of $L[q, \mathbf{Z}]$. Put $W=(\mathbf{Q} h)^{\perp}$ and let $\psi$ be the restriction of $\varphi$ to $W$. Then $L \cap W$ is maximal in $W$. Put $M=L \cap W$. Because \# $\left(S O^{\varphi}(V) \backslash S O^{\varphi}(V)_{\mathbf{A}} / C(L)\right)=1$, mass formula (2) in the introduction provides

$$
\# L[q, \mathbf{Z}]=[\Gamma(L): 1] \cdot \mathfrak{m}\left(S O^{\psi}(W), S O^{\psi}(W)_{\mathbf{A}} \cap C(L)\right),
$$

where $\Gamma(L)=S O^{\varphi}(V) \cap C(L)$. We see that $[\Gamma(L): 1]=2^{9} \cdot 3^{2} \cdot 5$ from $\left[O^{\varphi}(V) \cap D(L)\right.$ : $1]=2^{10} \cdot 3^{2} \cdot 5$, which is given by the proof of [5, Lemma 1.6]. Now, since $M$ is maximal in $W,(4)$ in the introduction combined with (5) gives

$$
\mathfrak{m}\left(S O^{\psi}(W), S O^{\psi}(W)_{\mathbf{A}} \cap C(L)\right)=\left[C(M): S O^{\psi}(W)_{\mathbf{A}} \cap C(L)\right] \mathfrak{m}\left(S O^{\psi}(W), C(M)\right) .
$$

Moreover applying Corollary 2.7 to $\mathfrak{m}\left(S O^{\psi}(W), C(M)\right)$, we see that

$$
\mathfrak{m}\left(S O^{\psi}(W), C(M)\right)=\left(2^{8} \cdot 3^{2} \cdot 5\right)^{-1}[\widetilde{M}: M]^{2} \prod_{p \mid \mathfrak{e}} \lambda_{p},
$$

where $\mathfrak{e}$ is the product of all prime numbers for which $\widetilde{M}_{p} \neq M_{p}$ and $\lambda_{p}$ is as in Corollary 2.7. From now on, we only treat the case of $q \equiv 2(\bmod 8)$, because the other cases can be handled in a similar way.

To compute $\left[C(M): S O^{\psi}(W)_{\mathbf{A}} \cap C(L)\right],[\widetilde{M}: M]$, and $\prod_{p \mid \mathfrak{e}} \lambda_{p}$, we need to determine the core dimension $s_{p}$ of $\left(W_{p}, \psi_{p}\right)$, the core dimension $t_{p}$ of $\left(V_{p}, \varphi_{p}\right)$ for any prime number $p$, the characteristic algebra $Q(\varphi)$ of $(V, \varphi)$, and the characteristic algebra $Q(\psi)$ of $(W, \psi)$. As for $t_{p}$, by $[8,(28.4)]$ we have $t_{p}=0$ if $p \equiv 1(\bmod 4)$, and $t_{p}=2$ if $p=2$ or $p \equiv 3$ $(\bmod 4)$. By the proof of $[\mathbf{8}$, Theorem 28.5$], Q(\varphi)$ is a definite quaternion algebra over 
$\mathbf{Q}$ ramified exactly at 2 . Now, assume $\left(\frac{-1}{p}\right)=1$, then $t_{p}=0$. Hence $s_{p}=1$, and then $p$ is unramified in $Q(\psi)$ by the definition. Next assume $\left(\frac{-1}{p}\right)=-1$. Let $K_{p}$ be the discriminant field of $\left(V_{p}, \varphi_{p}\right)$, that is, $K_{p}=\mathbf{Q}_{p}(\sqrt{-1})$. We have $t_{p}=2$, and hence $s_{p}=1$ or 3 according as $c^{-1} q \in \kappa_{p}\left[K_{p}^{\times}\right]$or $c^{-1} q \notin \kappa_{p}\left[K_{p}^{\times}\right]$. Here $c$ and $\kappa_{p}$ are as in the case $t=2$ in Section 2.3. Since $Q(\varphi)_{p}=M_{2}\left(\mathbf{Q}_{p}\right)$ is the Clifford algebra of the restriction of $\varphi_{p}$ to a core subspace of $V_{p}$, we have $c \in \kappa_{p}\left[K_{p}^{\times}\right]$as was mentioned in the case $t=2$ in Section 2.3. Then $c^{-1} q \in \kappa_{p}\left[K_{p}^{\times}\right]$if and only if $p \nmid q$ because $K_{p}$ is unramified over $\mathbf{Q}_{p}$. Therefore if $p \mid q$, then $s_{p}=3$, and hence $p$ is ramified in $Q(\psi)$ because $Q(\psi)_{p}$ is the even Clifford algebra of the restriction of $\psi_{p}$ to a core subspace of $W_{p}$. Also if $p \nmid q$, then $s_{p}=1$, and hence $p$ is unramified in $Q(\psi)$. Finally we consider the case of $p=2$. By [8, Theorem 26.6 (i)] the number of prime numbers ramified in $Q(\psi)$ is odd, since $Q(\psi)$ is ramified at the archimedean prime of $\mathbf{Q}$ from $[\mathbf{8},(28.3 \mathrm{~b})]$. From the above argument, $p$ is ramified in $Q(\psi)$ if and only if $p \mid q$ and $p \equiv 3(\bmod 4)$ for an odd prime number $p$. The number of these prime factors of $q$ is even because $q \equiv 2(\bmod 8)$. Therefore 2 must be ramified in $Q(\psi)$. From this we have $s_{2}=3$.

Once we obtain the data of $s_{p}, t_{p}, Q(\varphi)$, and $Q(\psi)$, Theorem 3.8 gives $[C(M)$ : $\left.S O^{\psi}(W)_{\mathbf{A}} \cap C(L)\right]=2^{\ell}$, where $\ell$ is the number of all odd prime factors of $q$. Applying $[\mathbf{8},(21.3 \mathrm{e})$ and Theorem 31.2 (iii) $]$ to $(W, \psi)$ and $M$, we find $[\widetilde{M}: M]=2 q$, since the discriminant field of $\psi$ is $\mathbf{Q}(\sqrt{q})$. By $[\mathbf{8},(21.3 \mathrm{~b})]$ we have $\mathfrak{e}=2 q$. Thus by Corollary 2.7 we see that $\prod_{p \mid \mathfrak{e}} \lambda_{p}=2^{-(\ell+3)} \cdot 3 \prod_{p \mid q}\left(1+\left(\frac{-1}{p}\right) \frac{1}{p^{2}}\right)$. Combining these with (4.9), (4.10), and (4.11), we obtain (4.4). Assertion (4.5) can be obtained in a similar way. This proves (i).

Assume $n=8$. Then by $\left[\mathbf{1 0}\right.$, Theorem 7.5] we have $L\left[q, 2^{-1} \mathbf{Z}\right] \neq \emptyset$ for any $q$. $[\mathbf{3}$, Section 5.16] shows that $\left[O^{\varphi}(V) \cap D(L): 1\right]=2^{14} \cdot 3^{5} \cdot 5^{2} \cdot 7$, and hence $[\Gamma(L): 1]=$ $2^{13} \cdot 3^{5} \cdot 5^{2} \cdot 7$. Moreover by $[8,(28.4)]$, for any prime number $p$ we have $t_{p}=0$. Thus $s_{p}=1$, and hence $Q(\psi)$ is unramified at $p$ for any $p$. Using these, we obtain (ii) in a similar way to the proof of (i).

Assume $n=10$. Then from [4, Section 6.8] we obtain $\left[O^{\varphi}(V) \cap D\left(L_{1}\right): 1\right]=2^{17} \cdot 3^{5}$. $5^{2} \cdot 7$ and $\mathfrak{m}\left(O^{\varphi}(V), D(L)\right)=\left(2^{18} \cdot 3^{5} \cdot 5 \cdot 7\right)^{-1}$. From these we have $\left[\Gamma\left(L_{1}\right): 1\right]=2^{16} \cdot 3^{5} \cdot 5^{2} \cdot 7$ and $\left[\Gamma\left(L_{2}\right): 1\right]=2^{17} \cdot 3^{4} \cdot 5^{2} \cdot 7$. Now $\left[\mathbf{1 0}\right.$, Theorem 7.5] leads that $L_{1}[q, \mathbf{Z}] \neq \emptyset$ if $q \equiv 1,2$ $(\bmod 4)$, and $L_{1}\left[q, 2^{-1} \mathbf{Z}\right] \neq \emptyset$ for any $q$. Taking $h \in L_{1}[q, \mathfrak{b}]$ with $\mathfrak{b}=\mathbf{Z}$ or $2^{-1} \mathbf{Z}$ according as $q \equiv 1,2(\bmod 4)$ or $q \equiv 3(\bmod 4)$, we define $(W, \psi)$ in the same manner as in the case $n=6$. Then by (2), (4), and (5) we obtain

$$
\frac{\# L_{1}[q, \mathfrak{b}]}{2^{16} \cdot 3^{5} \cdot 5^{2} \cdot 7}+\frac{\# L_{2}[q, \mathfrak{b}]}{2^{17} \cdot 3^{4} \cdot 5^{2} \cdot 7}=\left[C(M): S O^{\psi}(W)_{\mathbf{A}} \cap C(L)\right] \mathfrak{m}\left(S O^{\psi}(W), C(M)\right) .
$$

Here $M=L \cap W$, which is maximal in $W$. Then we can show (iii) in a similar way to the proof of (i). This completes the proof.

We note that (4.5), (4.6), and (4.8) can also be seen by applying [4, Theorem 1.5] to $(V, \varphi)$, a maximal lattice $L$ in $V$, and $q$ in each case, since $L[q]=L\left[q, 2^{-1} \mathbf{Z}\right]$. The fact $L[q]=L\left[q, 2^{-1} \mathbf{Z}\right]$ can be obtained as follows: Taking $h \in L[q]$, we have $\mathfrak{b} \subset \varphi(h, L) \subset$ $2^{-1} \mathbf{Z}$ from [10, Theorem 3.5], with $\mathfrak{b}$ of (4.2). Since $\mathfrak{b}=2^{-1} \mathbf{Z}$ in the case of (4.5), (4.6), or (4.8), we obtain $h \in L\left[q, 2^{-1} \mathbf{Z}\right]$, which proves the desired fact. 
On the sums of $n$ squares we remark that if $n=3$, then $\# L[q, \mathbf{Z}]$ is given by $[\mathbf{6}$, (12.10)]. Also if $n=5,7$, or 9 , then [6, Theorem 13.14] provides $\# L\left[q, 2^{-1} \mathbf{Z}\right]$ and $\# L[q, \mathbf{Z}]$. When $n=4$, by $\left[\mathbf{1 0}\right.$, Theorem 7.5] we have $\# L\left[q, 2^{-1} \mathbf{Z}\right]=\# L[q]$ if $q$ is odd, and $\# L[q, \mathbf{Z}]=\# L[q]$ if $q$ is even. The number $\# L[q]$ is given by [5, Section 1.7]. Here $L$ is a maximal lattice in $\mathbf{Q}_{n}^{1}$ and $q$ is a squarefree positive integer.

Let us give a few numerical examples for (4.7). Let $m(q)$ be the right-hand side of (4.7) for a squarefree positive integer $q$. Then, for example, $\# L_{1}[1, \mathbf{Z}]=2^{2}, \# L_{2}[1, \mathbf{Z}]=$ 0 , and $m(1)=\left(2^{14} \cdot 3^{5} \cdot 5^{2} \cdot 7\right)^{-1} ; \# L_{1}[2, \mathbf{Z}]=2^{2}$, \# $L_{2}[2, \mathbf{Z}]=2^{2} \cdot 5$, and $m(2)=$ $\left(2^{15} \cdot 3^{5} \cdot 5^{2} \cdot 7\right)^{-1} \cdot 17 ; \# L_{1}[58, \mathbf{Z}]=2^{3} \cdot 149 \cdot 8069, \# L_{2}[58, \mathbf{Z}]=2^{3} \cdot 3^{4} \cdot 5 \cdot 2969$, and $m(58)=\left(2^{14} \cdot 3^{5} \cdot 5^{2} \cdot 7\right)^{-1} \cdot 17 \cdot 353641 ; \# L_{1}[494, \mathbf{Z}]=\# L_{2}[494, \mathbf{Z}]=2^{7} \cdot 3^{3} \cdot 5 \cdot 181 \cdot 14281$ and $m(494)=\left(2^{10} \cdot 3^{2} \cdot 7\right)^{-1} \cdot 181 \cdot 14281$. These numbers were verified by Yoshio Hiraoka using a computer.

\section{References}

[1] W. T. Gan, J. P. Hanke and J.-K. Yu, On an exact mass formula of Shimura, Duke Math. J., 107 (2001), 103-133.

[2] T. Hiraoka, On the class number of the genus of $\mathbf{Z}$-maximal lattices with respect to quadratic form of the sum of squares, J. Math. Kyoto Univ., 46 (2006), 291-302.

[ 3 ] G. Shimura, An exact mass formula for orthogonal groups, Duke Math. J., 97 (1999), 1-66.

[4] G. Shimura, The number of representations of an integer by a quadratic form, Duke Math. J., 100 (1999), 59-92.

[5] G. Shimura, The representation of integers as sums of squares, Amer. J. Math., 124 (2002), 1059-1081.

[6] G. Shimura, Arithmetic and analytic theories of quadratic forms and Clifford groups, Mathematical Surveys and Monographs, 109, Amer. Math. Soc., 2004.

[ 7 ] G. Shimura, Integer-valued quadratic forms and quadratic Diophantine equations, Documenta Math., 11 (2006), 333-367.

[8] G. Shimura, Arithmetic of quadratic forms, Springer Monographs in Mathematics, Springer, New York, 2010.

[ 9 ] C. L. Siegel, Über die analytische theorie der quadratischen formen, Ann. of Math. (2), 36 (1935), 527-606.

[10] T. Yoshinaga, On the solutions of quadratic Diophantine equations, Documenta Math., 15 (2010), 347-385.

\author{
Manabu Murata \\ College of Science and Engineering \\ Ritsumeikan University \\ Kusatsu \\ Shiga 525-8577, Japan \\ E-mail: murata31@pl.ritsumei.ac.jp
}

\section{Takashi YoshinagA}

Department of Mathematics

Ritsumeikan University

Kusatsu

Shiga 525-8577, Japan

E-mail: tyt24349@pl.ritsumei.ac.jp 